\title{
Ti spiego il mio problema: un'indagine sulle competenze argomentative nella risoluzione di problemi matematici
}

\section{I explain my problem to you: a survey on argumentative skills in solving mathematical problems}

\author{
Monica Avenia \\ Docente di scuola primaria - Italia \\ 凶monica.avenia@gmail.com
}

Sunto / Diversi studiosi hanno di recente approfondito la competenza argomentativa applicata a processi di problem solving da parte degli studenti già dagli ultimi anni della scuola dell'infanzia. In questo articolo viene presentata un'indagine effettuata in una classe III primaria di Imola (Bologna) durante l'anno scolastico 2018/2019, avente come focus di interesse l'analisi dell'argomentazione durante la risoluzione di problemi. Ci si riferisce nello specifico al problem solving quale approccio alla risoluzione di tre problemi matematici prendendo come riferimento metodologico il Progetto $\mathrm{ArAl}$ e il Rally Matematico Transalpino, nei quali emerge l'importanza di tale approccio all'interno delle prassi didattiche in matematica, con attenzione al linguaggio specifico della disciplina e all'utilizzo dell'argomentazione per sviluppare e riconoscere i processi emotivi e metacognitivi tramite il ragionamento e la riflessione.

Parole chiave: problem solving; metacognizione; competenza argomentativa; vissuti emotivi; apprendimento tra pari.
Abstract / In recent years, several researchers have deeply analyzed the argumentative skills applied to students' problem solving processes since their last years of kindergarten. The article presents a survey carried out in a third-grade class of a primary school in Imola (near Bologna) during the 2018/2019 school year. It is focused on the analysis of the argumentation during the resolution of mathematical problems. We specifically refer to problem solving as an approach to the resolution of three math problems taking as methodological reference the Progetto ArAl and the Rally Matematico Transalpino. They both show the importance of this approach within mathematical education, with a particular attention to the specific language of the discipline and the use of argumentation to develop and recognize emotions and metacognitive processes through reasoning and reflection.

Keywords: problem solving; metacognition; argumentative skill; emotions; cooperative learning. 
La decisione, in questo articolo, di trattare l'impiego della competenza argomentativa durante la risoluzione di problemi matematici legati a un ambito specifico, parte dall'assunto che:

«[...] fare matematica è in prima istanza affrontare problemi, [...] la soluzione di un problema non sorge mai dal nulla; ma dipende sempre dall'esperienza di un soggetto. Dunque la soluzione dei problemi ( $\mathrm{o}$, come si dice anche in Italia, il problem solving) è una condizione ottimale per l'apprendimento».

(D'Amore, 1993, pp. 11-13)

Ci si è avvalsi dei principi del Progetto ArAl ${ }^{1}$ e del Rally Matematico Transalpino ${ }^{2}$ nei quali emerge l'importanza delle attività di problem solving all'interno delle prassi didattiche in matematica con attenzione al linguaggio specifico della disciplina e all'utilizzo dell'argomentazione per sviluppare un approccio metacognitivo nello studente, che scaturisce dal suo ruolo di protagonista attivo nel processo di apprendimento. Bisogna tenere conto che gli esercizi e i problemi concernono due differenti applicazioni didattiche in base allo scopo che l'insegnante vuole raggiungere e al tipo di attività richiesta all'allievo. Occorre fare una distinzione e maggior chiarezza tra esse, come esortano a fare in particolare due ricercatori:

\begin{abstract}
«Si ha un esercizio quando la risoluzione prevede che si debbano utilizzare regole e procedure già apprese, anche se ancora in corso di consolidamento; gli esercizi ritornano dunque nella categoria delle prove a scopo di verifica immediata o di rafforzamento; si ha invece un problema quando una o più regole o nozioni che dovrebbero essere utilizzate per la risoluzione non sono ancora bagaglio cognitivo del risolutore (alcune di esse potrebbero essere proprio in quell'occasione in via di esplicitazione); a volte è la successione stessa delle operazioni risolventi a richiedere un atto strategico, talvolta creativo, da parte del risolutore».
\end{abstract}

(D’Amore \& Sbaragli, 2011, p. 95)

Da questa distinzione si evince come non sia il testo in sé a costituire un esercizio o un problema ma un complesso legato a situazioni didattiche, a capacità individuali, a fattori emotivi o più semplicemente al "contratto" che si è stabilito tra insegnante e alunno. Partendo dal presupposto che anche gli esercizi hanno la loro valenza in ambito didattico, soprattutto se utilizzati per consolidare conoscenze acquisite, in questa indagine ci si occuperà principalmente di una matematica che passa attraverso la risoluzione di problemi, dal risultato non predeterminato, che ammettono diverse strategie risolutive e diverse modalità di far fronte al testo proposto; questi problemi prevedono tipicamente I'accompagnamento di argomentazioni approfondite ed esplicative del percorso intrapreso al fine di rendere minore la distanza percepita da ogni allievo tra il linguaggio matematico e gli altri linguaggi di uso comune.

Quando uno studente si trova di fronte a un problema, si può dire che si trova dinanzi a una situazione problematica, e vive un momento di apprendimento che ne comporta sì la risoluzione ma non con una semplice ripetizione di conoscenze: c'è la necessità di formulare ipotesi nuove.

Nel campo del problem solving, tra numerosi altri studi, è stato molto importante il contributo dell'opera How to solve it (Polya, 1945), in cui viene enfatizzata l'importanza dei problemi nell'attività matematica evidenziando quattro fasi principali di risoluzione:

1. Per approfondimenti si rinvia al sito: www.progettoaral.it

2. Da ora in poi verrà citato con l'abbreviazione RMT. Per approfondimenti si rinvia al sito: https://armtint.eu 
- capire il problema cercando di porsi alcune domande;

- ideare un piano per trovare la relazione tra dati e incognita;

- eseguire il piano;

- ritornare indietro per verificare il procedimento e controllare i risultati per poi discuterne apertamente.

La raccomandazione che Polya dà agli insegnanti è quella di percorrere in un certo senso l'approccio costruttivista dell'apprendimento andando a enfatizzare l'importanza del ruolo dell'allievo nella costruzione del sapere. Propone infatti di distaccarsi da compiti meramente esecutivi a favore di esperienze significative e stimolanti che aumentino la motivazione ad apprendere.

\subsection{Valenza didattica dell'argomentazione}

Costruire, sviluppare, promuovere, rafforzare e avvalersi della motivazione è una delle più serie sfide che la scuola deve fronteggiare. La motivazione muove l'alunno a spingersi verso l'apprendimento. Entrando nello specifico dell'argomento, come sostengono D'Amore e Marazzani:

«[...] nell'insieme delle capacità che si devono mettere in atto per risolvere un problema, anche la capacità di stare attenti è fondamentale [...] motivare tanto da far sì che il bambino guardi il testo del problema con attenzione e curiosità, fa parte della professionalità dell'educatore».

(D’Amore \& Marazzani, 2011, p. 54)

Un consiglio pratico che viene dato dagli autori è quello, ad esempio, di fissare tra i criteri di valutazione il ragionamento che l'allievo ha seguito nella risoluzione di un problema piuttosto che valutare con estrema pignoleria la mera esecuzione dei calcoli. Ciò potrebbe giovare all'incremento di motivazione in quanto ad essa è strettamente correlato il lato affettivo ed emotivo dei bambini, bambini che si mettono in gioco e che potrebbero manifestare sensazioni negative creando, successivamente, situazioni di blocco di fronte a possibili difficoltà anziché cercare di superare l'ostacolo. Nella manifestazione della motivazione, favorita dall'insegnante con determinate pratiche didattiche di tipo attivo, I'allievo è il protagonista della costruzione del proprio sapere e della propria conoscenza.

Citando Di Martino (2017, p. 25), ci viene ricordato che «dal punto di vista epistemologico, infatti, molti matematici sottolineano come l'essenza del fare matematica sia il risolvere problemi» e inoltre «-sconfinando negli aspetti didattici - che il miglior modo per imparare a risolvere problemi sia affrontare problemi».

Anche dal punto di vista normativo, la lettura attenta delle Indicazioni Nazionali italiane per la scuola dell'infanzia e il primo ciclo (Ministero dell'Istruzione, dell'Università e della Ricerca [MIUR], 2012) evidenzia come lo sviluppo di competenze argomentative e nell'ambito del problem solving sia un traguardo fondamentale di tutta l'educazione matematica dai 3 ai 14 anni: un traguardo dunque da sviluppare in verticale, ma anche trasversale alle diverse discipline e soprattutto che assume un ruolo cruciale nella formazione del cittadino adulto.

I traguardi delle Indicazioni Nazionali suggeriscono significativi contesti di lavoro riferiti alla scienza, alla tecnologia, alla società. La matematica, tuttavia, permette di sviluppare competenze trasversali importanti attraverso attività che valorizzano i processi tipici della disciplina: «[...] in particolare, la matematica [...] contribuisce a sviluppare la capacità di comunicare e discutere, di argomentare in modo corretto, di comprendere i punti di vista e le argomentazioni degli altri» (MIUR, 2012, p. 49). Tali competenze sono rilevanti per la formazione di una cittadinanza attiva e consapevole, in cui ogni persona è disponibile all'ascolto attento e critico dell'altro e a un confronto basato sul riferimento ad argomenti pertinenti e rilevanti.

Il laboratorio di matematica rappresenta, in questo scenario, un contesto naturale per stimolare le capacità di argomentare e favorire il confronto fra pari: 
«[...] in matematica, come nelle altre discipline scientifiche, è elemento fondamentale il laboratorio, inteso sia come luogo fisico, sia come momento in cui l'alunno è attivo, formula le proprie ipotesi e ne controlla le conseguenze, progetta e sperimenta, discute e argomenta le proprie scelte, impara a raccogliere dati, negozia e costruisce significati, porta a conclusioni temporanee e a nuove aperture la costruzione delle conoscenze personali e collettive».

(MIUR, 2012, p. 49)

Alla luce della descrizione che ne viene data nelle Indicazioni Nazionali, il laboratorio può costituire anche una palestra per imparare a fare scelte consapevoli, a valutarne le conseguenze e quindi ad assumersene la responsabilità, aspetti anche questi centrali per l'educazione a una cittadinanza attiva. Tutto ciò perché viene data, a livello normativo, estrema importanza allo sviluppo di un'adeguata visione della matematica, non ridotta a un insieme di regole da memorizzare e applicare, ma riconosciuta e apprezzata come contesto per porsi e affrontare problemi significativi.

Problem solving e argomentazione costituiscono dunque ufficialmente il leitmotiv di tutta l'educazione matematica dello studente.

Problem solving e argomentazione, inoltre, sono tra loro collegati: per valutare la risoluzione di un problema, dobbiamo avere informazioni sia sui processi attivati (quindi è necessaria la spiegazione), sia valutare le giustificazioni delle scelte fatte (quindi la vera e propria argomentazione). D'altra parte, richiedere di argomentare ha senso laddove lo studente è chiamato a fare delle scelte, ad assumersi delle responsabilità nell'attivazione dei processi di pensiero.

È necessaria, dunque, una didattica attenta alla visione della matematica che gli allievi si costruiscono, cercando di ridurre il più possibile quelli che Zan (2007) definisce "i danni del bravo insegnante", danni che si presentano nel momento in cui si predispongono piani di apprendimento secondo schemi tradizionali e predefiniti. C'è invece la necessità di provare a fondare il proprio agire didattico partendo dal vedere i possibili errori degli allievi anche come opportunità di scambio aperto e crescita condivisa.

\subsection{Progetto ArAl e Rally Matematico Transalpino: due realtà concrete di utilizzo della compe- tenza argomentativa}

Il Progetto ArAl e il RMT sono due realtà, due metodologie di insegnamento concrete e attuali che si approcciano alla matematica in modo innovativo e che fanno dell'argomentazione uno dei capisaldi metodologici.

Il progetto ArAl si colloca all'interno di quella cornice teorica che assume la denominazione di Early Algebra, un'area di ricerca nell'ambito dell'educazione matematica che promuove l'insegnamento dell'aritmetica in una prospettiva algebrica sin dai primi anni della scuola primaria. ${ }^{3}$

Punto di forza e di partenza dell'Early Algebra è l'attenzione verso la semantica del linguaggio algebrico e la volontà di introdurlo fin da subito affiancato al linguaggio naturale, cosicché gli alunni possano costruire quelli che sono i modelli mentali propri di tale linguaggio. Si parla a tal proposito di "balbettio algebrico", intendendo con questo termine quanto definito da Malara e Navarra (2008, pp.168-169): «l'appropriazione sperimentale di un nuovo linguaggio, nel quale le regole trovino la loro collocazione gradualmente, all'interno di un contratto didattico adatto e tollerante». È a partire da questi presupposti che il progetto ArAl sviluppa l'attenzione verso le proposte, le affermazioni, le sperimentazioni degli alunni stessi, facendo partire l'indagine e lo studio dei fenomeni della matematica, accompagnando gli allievi verso una comprensione radicata tramite una discussione collettiva sui temi matematici. Attraverso questo approccio gli alunni sono portati a «riflettere continuamente sulle idee, sulle opinioni, sugli errori e sui successi conseguiti» (Malara, 2009, p. 18); a riflettere quindi sui processi, a porsi in relazione con le ipotesi e con le proposte dei compagni, a confrontarle e classificar-

3. La scuola primaria in Italia dura cinque anni e corrisponde alla scuola elementare nel Canton Ticino. 
le, a valutare le loro proprie convinzioni, a operare scelte consapevoli. Tutto ciò si deve poi misurare con la competenza dell'insegnante nel gestire tale situazione e nel permettere che da essa gli alunni possano trarre il maggior giovamento possibile. Attraverso la verbalizzazione e l'argomentazione, gli alunni possono essere avvicinati più facilmente alla comprensione dell'aritmetica in chiave algebrica. Per sviluppare il linguaggio della matematica, il progetto ArAl spinge gli allievi a discutere, ascoltare, proporre, motivare affermazioni ed esplicitare idee sui temi matematici trattati. Questo approccio didattico si pone in maniera trasversale in tutti i gradi di scuola, e l'insegnante che vuole intraprendere questo percorso è costantemente seguito oltre ad avere diverse possibilità di formazione.

II RMT, nato nel 1992 in Svizzera, invece, si configura come un confronto fra classi, dalla terza primaria al secondo anno di scuola secondaria di secondo grado, ${ }^{4}$ nell'ambito della risoluzione di problemi di matematica. Propone situazioni problematiche per le quali non si dispone di una soluzione immediata e che conducono a inventare una strategia, a fare tentativi, a congetturare, a verificare, a giustificare la soluzione, a spiegare le proprie procedure. Tra i suoi obiettivi, vi è la pratica del ragionamento scientifico: sviluppo dell'autonomia di apprendimento, organizzazione di una ricerca, rigore delle notazioni, capacità di argomentare e di comunicare i risultati.

II RMT non è solo una gara, è anche l'occasione per un approfondito lavoro di analisi didattica.

Per quanto concerne il progetto ArAl, in questa indagine sono stati utilizzati alcuni traguardi per lo sviluppo delle competenze (che a loro volta si rifanno ai traguardi delle Indicazioni Nazionali) incentrati sull'utilizzo del linguaggio matematico ponendo l'attenzione alla discussione di classe. Dal RMT sono stati scelti i tre problemi somministrati al gruppo classe applicandone la metodologia di svolgimento. È evidente come entrambi gli approcci presentino elementi innovativi rispetto all'apprendimento della matematica. Si riscontrano molti punti in comune: entrambi si propongono

«[...] di fare matematica attraverso la risoluzione di problemi, [...] di sviluppare la capacità di lavorare in gruppo sentendosi responsabili, di imparare a parlare di matematica. [...] II confronto fra gli insegnanti sulla progettazione delle attività di problem solving e sui processi risolutivi messi in atto dagli allievi si configura come una significativa modalità di auto-formazione».

(Zan, 2007, pp. 268-271)

Un ulteriore obiettivo di fondo dei due approcci è quello di offrire agli insegnanti spazi per la riflessione sulle loro conoscenze e sul loro agire didattico in aula, affinché gli alunni possano sviluppare processi cognitivi e competenze sociali in sinergia.

I bambini, protagonisti del loro sapere, hanno la possibilità concreta di esperire in classe un modello di didattica incentrata sugli aspetti di una vita societaria democratica, ampliando così l'acquisizione delle competenze trasversali previste dalla Comunità Europea.

\section{Sperimentazione in aula}

Questa sperimentazione, attraverso un primo questionario a risposta aperta, è volta a esplorare in un primo momento le convinzioni dei bambini sul concetto di problema, sul suo utilizzo e sull'utilità estrinseca che esso ha per ciascun bambino, nonché le eventuali difficoltà che sorgono nel momento in cui un bambino si trova a riflettere individualmente sulle esperienze avute, prima della sperimentazione, durante la risoluzione di problemi matematici. La sperimentazione, per come è stata struttu-

4. La scuola secondaria di secondo grado in Italia dura cinque anni e corrisponde all'ultimo anno di scuola media e alla scuola media superiore o scuole professionali nel Canton Ticino. 
rata, si propone di fornire gli strumenti necessari per una chiave interpretativa funzionale alla verifica delle ipotesi di ricerca.

In un secondo momento, dopo la somministrazione dei problemi matematici da svolgere in gruppo, argomentando la risoluzione e la procedura adottata, questo lavoro indaga le dinamiche argomentative e cooperative che si sono create in contesto gruppale durante lo svolgimento del compito.

Infine, tramite la somministrazione di un questionario individuale finale, vengono raccolti i vissuti e le impressioni dei bambini, si analizza come le strategie argomentative applicate ai problemi possano migliorare le competenze linguistiche, nonché facilitare i processi comunicativi in un contesto cooperativo e chiarire quelli cognitivi che si attuano durante la fase di svolgimento del compito assegnato. Di seguito sono riportati gli obiettivi di apprendimento che si intende far raggiungere, attraverso la sperimentazione, agli allievi e sui quali sono costruiti gli indicatori dei questionari somministrati. Tali obiettivi fanno riferimento alle Indicazioni Nazionali 2012 e agli obiettivi specifici del progetto ArAl. I traguardi per lo sviluppo delle competenze al termine della classe $\mathrm{V}$ primaria presi come riferimento dalle Indicazioni Nazionali (MIUR, 2012) sono i seguenti:

- Legge e comprende testi che coinvolgono aspetti logici e matematici.

- Riesce a risolvere facili problemi in tutti gli ambiti di contenuto, mantenendo il controllo sia sul processo risolutivo, sia sui risultati. Descrive il procedimento seguito e riconosce strategie di soluzione diverse dalla propria.

- Costruisce ragionamenti formulando ipotesi, sostenendo le proprie idee e confrontandosi con il punto di vista di altri.

Gli obiettivi del progetto ArAl $^{5}$ relativi a strategie di problem solving considerati per strutturare il questionario, con particolare riguardo all'utilizzo del linguaggio matematico nell'argomentazione durante la risoluzione di un problema, sono di seguito riportati:

Categoria Numeri:

- Usare termini specifici del linguaggio matematico.

- Eseguire mentalmente semplici operazioni con le quattro operazioni anche esplicitando strategie e proprietà usate.

Categoria Relazioni numeri dati e previsioni:

- In riferimento a semplici situazioni problematiche, distinguere i dati dai loro valori numerici, assegnando o facendo variare tali valori.

- Oggettivare le relazioni tra i dati in semplici situazioni problematiche.

- Utilizzare le relazioni individuate per prevedere nuovi risultati sulla base di quelli precedenti.

- Giustificare la procedura usata.

Nelle Tabelle 1 e 2, riportate nel prossimo paragrafo, è possibile visualizzare come sono stati strutturati i questionari a partire dagli indicatori individuati.

\subsection{Metodologia}

In questo lavoro, ci si è avvalsi di una metodologia che riflette una ricerca di carattere qualitativo. La sperimentazione descritta in questa esperienza didattica si è svolta in una classe terza della scuola primaria "Athos Rubri" afferente all'Istituto Comprensivo n. 6 di Imola. Nell'indagine sono stati 
coinvolti 21 bambini su un totale di $22^{6}$ tra cui 10 maschi e 11 femmine. Tra marzo e aprile 2019, in accordo con l'insegnante della classe, si sono fissati cinque appuntamenti a cadenza settimanale, per un totale di 15 ore di lavoro con gli studenti.

La sperimentazione in classe si è articolata come segue:

1. Nel primo incontro sono stati presentati agli alunni i passaggi della sperimentazione ed è stato somministrato il questionario in entrata (Tabella 1). II questionario è composto da 10 domande riferite a quattro indicatori al fine di «portare alla luce le convinzioni [...] sulla visione della matematica» (Zan, 2007, p. 223) che l'allievo sta costruendo, nello specifico sui problemi che sono entrati a far parte della routine del suo percorso scolastico.

\begin{tabular}{|c|c|c|c|c|}
\hline Indicatori & $\begin{array}{l}\text { Indagare le concezio- } \\
\text { ni spontanee sul } \\
\text { concetto di problema }\end{array}$ & $\begin{array}{l}\text { Analizzare le } \\
\text { strategie attuate per } \\
\text { la risoluzione di un } \\
\text { problema matematico }\end{array}$ & $\begin{array}{l}\text { Indagare i vissuti } \\
\text { emotivi che emergo- } \\
\text { no durante la } \\
\text { risoluzione di un } \\
\text { problema matematico }\end{array}$ & $\begin{array}{l}\text { Indagare le compe- } \\
\text { tenze argomentative } \\
\text { attuate per spiegare } \\
\text { la procedura di } \\
\text { risoluzione di un } \\
\text { problema matematico }\end{array}$ \\
\hline Domande & $\begin{array}{l}\text { 1) Che cos'è per te un } \\
\text { problema di matema- } \\
\text { tica? } \\
\text { 2) Quali caratteristiche } \\
\text { ha per te un problema } \\
\text { di matematica? } \\
\text { 3) A cosa serve, per te } \\
\text { risolvere un problema? }\end{array}$ & $\begin{array}{l}\text { 4) Cosa fai quando } \\
\text { risolvi un problema? } \\
\text { 5) Qual è per te la } \\
\text { difficoltà che ti trovi } \\
\text { ad affrontare? }\end{array}$ & $\begin{array}{l}\text { 6) Cosa provi quando } \\
\text { devi risolvere un } \\
\text { problema? } \\
\text { 7) Da } 1 \text { a } 5 \text { quanto ti } \\
\text { senti a tuo agio nel } \\
\text { risolvere un problema? } \\
\text { Perché? }\end{array}$ & $\begin{array}{l}\text { 8) Di solito spieghi } \\
\text { come sei giunto alla } \\
\text { soluzione di un } \\
\text { problema? } \\
\text { 9) In quali situazioni ti } \\
\text { capita solitamente? } \\
\text { Descrivi un episodio. } \\
\text { 10) Durante la corre- } \\
\text { zione ti confronti con i } \\
\text { tuoi compagni e/o con } \\
\text { la maestra? Se sì, } \\
\text { come? }\end{array}$ \\
\hline
\end{tabular}

Tabella 1. Questionario in entrata.

2. Tre incontri sono stati incentrati sulla risoluzione di alcuni problemi selezionati dal RMT. I problemi scelti sono riportati nei prossimi paragrafi, insieme a un'analisi a priori del loro svolgimento e delle loro principali caratteristiche. Prima della risoluzione sono state descritte ai bambini le modalità di lavoro.

Con l'aiuto dell'insegnante, la classe è stata divisa in 7 gruppi omogenei composti da 3 bambini, gruppi rimasti fissi per l'intera fase della sperimentazione. Ogni gruppo aveva a disposizione 50 minuti per svolgere il problema proposto e poteva avvalersi degli strumenti che riteneva necessari per la risoluzione. È stato posto l'accento sull'importanza di trascrivere e argomentare il percorso fatto per giungere a una o più strategie di risoluzione dei problemi matematici.

3. Al termine del quinto incontro è stato somministrato il questionario in uscita sull'esperienza fatta. Questo dopo aver presentato all'intera classe i protocolli di risoluzione dei problemi dei vari gruppi in modo da avviare una discussione di classe sulle diverse strategie di risoluzione trovate e ampliare il bagaglio di competenze di ogni singolo allievo, discussione che l'insegnante ha proseguito anche a posteriori. II questionario era strutturato in nove domande che fanno riferimento a quattro indicatori pensati per analizzare le impressioni e i vissuti emotivi scaturiti dall'esperienza delle modalità di risoluzione dei problemi del RMT, focalizzando l'attenzione sull'importanza dell'argomentazione come supporto alle strategie di risoluzione.

6. Un alunno certificato non ha potuto partecipare alla sperimentazione per problemi di salute. 


\begin{tabular}{|c|c|c|c|c|}
\hline Indicatori & $\begin{array}{l}\text { Indagare le concezio- } \\
\text { ni personali sulla } \\
\text { somministrazione di } \\
\text { problemi matematici } \\
\text { seguendo la metodo- } \\
\text { logia del RMT }\end{array}$ & $\begin{array}{l}\text { Individuare la } \\
\text { ripartizione dei ruoli } \\
\text { per risolvere il } \\
\text { problema matematico } \\
\text { in contesto gruppale }\end{array}$ & $\begin{array}{l}\text { Evidenziare le } \\
\text { strategie argomenta- } \\
\text { tive messe in atto per } \\
\text { formulare la procedu- } \\
\text { ra di risoluzione del } \\
\text { problema }\end{array}$ & $\begin{array}{l}\text { Rilevare i vissuti dei } \\
\text { bambini durante la } \\
\text { risoluzione in gruppo } \\
\text { dei problemi } \\
\text { matematici }\end{array}$ \\
\hline Domande & $\begin{array}{l}\text { 1) Quali differenze hai } \\
\text { trovato mentre svolgevi } \\
\text { i problemi del RMT } \\
\text { proposti rispetto a quelli } \\
\text { svolti in modo } \\
\text { tradizionale? } \\
\text { 2) Se hai qualcosa da } \\
\text { aggiungere in merito ai } \\
\text { problemi del RMT puoi } \\
\text { scriverlo di seguito. }\end{array}$ & $\begin{array}{l}\text { 3) Durante lo svolgi- } \\
\text { mento del problema in } \\
\text { gruppo come vi siete } \\
\text { divisi i compiti? } \\
\text { 4) Qual è stato il tuo } \\
\text { ruolo durante la } \\
\text { risoluzione del } \\
\text { problema? }\end{array}$ & $\begin{array}{l}\text { 5) In che modo siete } \\
\text { arrivati alla risoluzione } \\
\text { del problema? Spiega le } \\
\text { fasi. } \\
\text { 6) Pensi sia utile } \\
\text { spiegare scrivendo, } \\
\text { tutto il percorso fatto } \\
\text { per arrivare alla } \\
\text { soluzione? Perché? }\end{array}$ & $\begin{array}{l}\text { 7) Quali sono le cose } \\
\text { positive per te, nello } \\
\text { svolgere un problema } \\
\text { secondo le regole del } \\
\text { RMT? } \\
\text { 8) Da } 1 \text { a } 5 \text { quanto ti sei } \\
\text { sentito a tuo agio nel } \\
\text { risolvere i tre problemi } \\
\text { presentati? Perché? } \\
\text { 9) Quali sono le } \\
\text { emozioni che hai } \\
\text { provato mentre hai } \\
\text { risolto i problemi con i } \\
\text { tuoi compagni di } \\
\text { classe? }\end{array}$ \\
\hline
\end{tabular}

Tabella 2. Questionario in uscita

\subsection{Problema n. 1 - Caccia al tre}

Il seguente problema è tratto dal RMT: 10.I.03; afferisce all'ambito NU (numeri), ed è somministrabile alle categorie: ${ }^{3} 3,4,5$.

«Isidoro sta scrivendo la successione dei numeri a partire da 1 :

$1,2,3,4,5,6,7,8,9,10,11,12, \ldots$

Ad un certo punto Isidoro scrive la cifra 3 per la venticinquesima volta.

Quale numero sta scrivendo Isidoro a quel punto?

Mostrate come l'avete trovato».

Questo problema fa riferimento alle conoscenze sul sistema di numerazione decimale posizionale. In questo problema occorre elencare la successione dei numeri, oppure solo i numeri contenenti la cifra 3 e fermarsi al numero 131 che la contiene per la venticinquesima volta.

Lo studente deve aver compreso che la cifra 3 va considerata qualunque sia il suo valore posizionale e che essa compare regolarmente nella posizione delle unità una volta in ogni decina, 10 volte (da 30 a 39) nella posizione delle decine in ogni centinaio, 100 volte (da 300 a 399) nella posizione delle centinaia in ogni migliaio e così via.

Gli errori che si possono commettere sono diversi:

1. i bambini non distinguono le due cifre 3 contenute nel numero 33 con la conseguenza di dare 132 come risposta al problema;

2. lacune nella numerazione per disattenzioni o imprecisioni che portano a una costruzione errata della successione dei numeri;

3. considerare solo la cifra 3 quando ha valore di unità e non di decina;

4. moltiplicare 3 per 25 , influenzati dall'interpretazione del numero ordinale "venticinquesima volta", riportata nell'enunciato, come se fosse "venticinque volte";

7. Le classi che partecipano al RMT si suddividono in otto categorie. Tre per la scuola primaria: cat.3 - classe terza, cat.4 - classe quarta, cat. 5 - classe quinta. Tre per la scuola secondaria di primo grado: cat.6 - classe prima, cat.7 - classe seconda, cat. 8 classe terza. Due per la scuola secondaria di secondo grado: cat.9 - classe prima, cat.10 - classe seconda. 
5. scrivere la cifra 3 ripetutamente per 25 volte per lettura errata del testo.

Gli errori degli studenti nella risoluzione del problema si possono ricondurre all'influenza in campo matematico delle competenze linguistiche in loro possesso e all'assenza totale del controllo del senso del testo stesso (soprattutto nei casi 4 e 5 sovraesposti), ma sono altresì rivelatori della confusione cifra-numero (ad esempio nel caso 1).

\subsection{Problema n. 2 - Tiro al barattolo}

II seguente problema è tratto dal RMT: 26.I.01; afferisce all'ambito OPN (operazioni aritmetiche con numeri naturali), ed è somministrabile alle categorie: 3,4 .

«In questo gioco di abilità bisogna far cadere uno dei quattro barattoli che sono appoggiati su un tavolo, lanciando una palla.

Quando un barattolo cade, si ottiene il numero di punti che è scritto sul barattolo e si rimette il barattolo al suo posto. Se nessun barattolo cade, non si ottengono punti.

Si guadagna un bell'orso di pelouche se si ottengono esattamente 32 punti, né più né meno, dopo aver lanciato cinque volte la palla.

Quali sono i barattoli che si devono far cadere per vincere l'orso lanciando cinque volte la palla? Indicate tutte le possibilità: quali barattoli dovranno cadere e quante volte ognuno di essi cadrà».

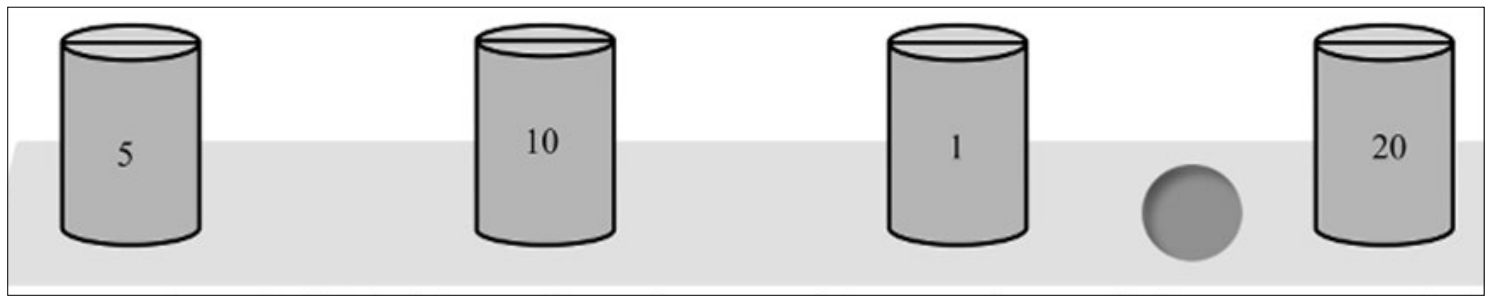

Figura 1. Barattoli con punteggi.

Questo problema riguarda le operazioni aritmetiche con numeri naturali. II compito degli studenti è scegliere l'operazione o le operazioni da utilizzare per la risoluzione, tenere conto delle loro proprietà, eseguire i calcoli corrispondenti. Dal punto di vista didattico questi problemi consentono di rafforzare il controllo delle relazioni e delle operazioni sui numeri naturali.

Allo studente viene richiesto di trovare tutte le addizioni di cinque termini scelti tra i numeri $0,1,5$, 10, 20 e la cui somma sia 32.

Nello specifico il ragionamento del gruppo deve analizzare le seguenti situazioni:

- capire che ad ogni lancio si possono ottenere 5, 10, 1, 20 o anche 0 punti;

- capire che la palla è lanciata cinque volte, che il numero di punti ottenuti è la somma di cinque termini presi tra i numeri precedenti e che uno stesso numero può essere ripetuto più volte.

Le diverse strategie possibili si possono riassumere come segue:

- simulare il gioco scegliendo il barattolo abbattuto o l'assenza di barattoli abbattuti ad ogni lancio, calcolare il numero di punti ottenuti e confrontarlo con 32 e, se necessario, ricominciare con la simulazione;

- fare dei tentativi addizionando cinque numeri tra 0, 1, 5, 10, 20 con ripetizione possibile e confrontare la somma con 32;

- capire che, per ottenere 32 punti, bisogna necessariamente far cadere due volte il barattolo con il numero 1 e che poi bisogna fare 30 con tre lanci con gli altri numeri. Procedere per tentativi o iniziare una ricerca sistematica: 
a. con presenza del numero 20: ottenere le due possibilità $20+10+0$ e $20+5+5$;

b. senza il numero 20 : ottenere la sola possibilità $10+10+10$;

- concludere che ci sono tre possibilità con gli addendi non disposti necessariamente in quest'ordine:
a. $20+10+1+1+0$;
b. $20+5+5+1+1$;
c. $10+10+10+1+1$.

Gli errori in cui gli alunni possono incorrere sono diversi:

- i bambini non considerano la possibilità di un lancio a vuoto a cui attribuire il punteggio 0 perché non rientra nello schema visivo della figura che accompagna il testo del problema;

- il bambino potrebbe considerare valida la somma aritmetica $20+10+1+1$, utilizzando quattro dei cinque tiri a disposizione, e ritenerla giusta perché si sono utilizzati meno tiri di quelli a disposizione;

- il bambino potrebbe non considerare che un barattolo può essere colpito più volte credendo che deve necessariamente colpirli tutti.

Gli errori degli studenti nella risoluzione di questo problema si possono ricondurre a una confusione sul controllo del senso del testo, seppure il testo sia costruito su una narrazione verosimile «dove la verosimiglianza narrativa riguarda anche gli scopi dei personaggi» (Zan, 2012a, p. 122).

\subsection{Problema n. 3 - Il cuore di Martina}

II seguente problema è tratto dal RMT: 22.I.09; afferisce all'ambito GP (geometria piana), ed è somministrabile alle categorie: 5, 6 .

\footnotetext{
«Martina ha fatto un disegno a forma di cuore sul suo quaderno.

Ha colorato il cuore di rosso e di azzurro la parte rimanente del quadrato.

Qual è la parte più grande, quella colorata in rosso o quella colorata in azzurro?

Spiegate come avete trovato la vostra risposta».
}

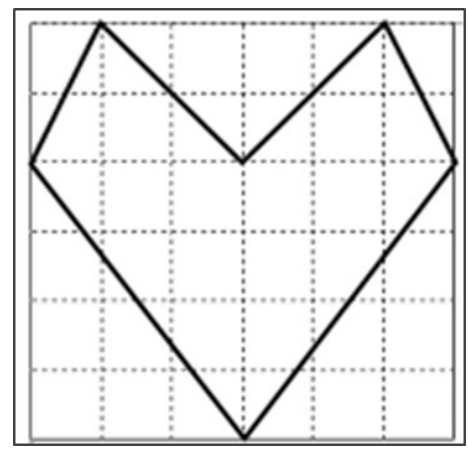

Figura 2. Cuore di Martina.

Il compito consiste nel confrontare delle aree di figure senza ricorrere al calcolo con le formule tradizionali, dato che i bambini del livello scolastico a cui viene somministrato il problema non conoscono ancora le formule per il calcolo dell'area. Si aiuteranno quindi tramite la scomposizione in unità determinate dalla "griglia" sulla quale le figure sono disegnate.

Per la risoluzione del problema è necessario confrontare l'area interna ed esterna di un poligono disegnato su una griglia quadrettata $6 \times 6$. II poligono ha un asse di simmetria e i suoi vertici si trovano sulle intersezioni della quadrettatura.

È necessario decontestualizzare la situazione: passare dall'immagine del "cuore in rosso" e del "resto 
del quadrato in azzurro" alle figure geometriche e capire che l'espressione "la parte più grande" si riferisce alle aree delle figure.

Si procede così a scomporre, mentalmente o con il disegno dei segmenti, le due forme in figure più semplici o elementari: quadretti della griglia quadrata o triangoli.

Fatta la scomposizione si passa al conteggio dei quadrati della griglia. Infine, è possibile effettuare le addizioni corrispondenti e confrontare le aree totali.

Tra i saperi mobilizzati nell'ambito geometrico rientrano: il concetto di unità d'area (quadretti della griglia), l'equivalenza e la trasformazione di unità d'area, la scomposizione o ricomposizione di parti di quadretti della griglia per spostamenti (traslazione o rotazione), il calcolo dell'area del rettangolo sulla griglia. In ambito aritmetico invece i saperi concernono il conteggio e l'addizione.

Si può procedere alla risoluzione tramite diverse procedure:

- Per i triangoli: la parte inferiore è scomposta in due rettangoli $3 \times 4$ suddivisi entrambi in due triangoli rettangoli dal contorno della figura.

- La parte superiore è scomposta in due quadrati centrali $2 \times 2$ e due rettangoli a destra e a sinistra del tipo $1 \times 2$; ciascuno di questi poligoni risulta suddiviso in due triangoli rettangoli dal contorno della figura. La spiegazione può presentarsi sotto forme differenti a seconda di come i bambini argomentano la risoluzione del problema.

- Con conteggio dei quadretti della griglia: sono presi in conto i quadretti interi e le parti di quadretto sono raggruppate per ricostituire dei quadretti interi.

Ciascuna di queste procedure può presentarsi con varianti molto diverse.

Nelle procedure per conteggio dei quadretti della griglia, gli ostacoli risiedono nella ricomposizione delle parti di quadretti. Per la parte superiore, il contorno del cuore divide i quadretti e i semi-quadretti triangolari o in quarti di quadretti triangolari o in tre quarti di quadretti a forma di trapezio; il compito di ricomposizione è facilitato in questo caso.

Nella parte inferiore dove il contorno segue la diagonale dei rettangoli di tipo $3 \times 4$ e divide sei quadretti in triangoli e trapezi di tre grandezze differenti, il conteggio dei quadretti può condurre di conseguenza a errori mentre i calcoli d'area effettuati sulla base di misure in $\mathrm{cm}$ e $\mathrm{mm}$ sono sempre inefficaci.

\section{Questionario in entrata: analisi dei dati rilevati}

Le risposte per esteso al questionario, riportate dagli alunni, sono consultabili nell'Allegato 1. Le risposte simili date dagli alunni sono state accorpate sotto un unico argomento relativo ai diversi indicatori al fine della misurazione statistica. II numero dei bambini menzionato si riferisce sempre in rapporto al totale degli alunni partecipanti che corrisponde a 21.

\subsection{Indagare le concezioni spontanee sui problemi}

Le domande riferite al primo indicatore "Indagare le concezioni spontanee sul concetto di problema matematico" (si veda la Tabella 1) hanno spinto gli alunni a riflettere su cos'è per loro un problema matematico, sullo scopo che ha e le caratteristiche che lo definiscono.

Per 7 bambini un problema viene definito tale quando il testo richiede delle «operazioni da trovare», simile all'affermazione «un testo con una domanda a cui dare una risposta», pensiero condiviso da altri 4 alunni e riconfermato nella domanda n. 2, quando tra le caratteristiche più condivise rientrano: domande a cui rispondere con operazioni (10 bambini), lettura del testo diverse volte per comprendere la consegna (4 bambini) al fine di individuare una domanda e una risposta ( 3 alunni). Come 
sostengono D'Amore e Sbaragli (2011, pp. 26-27), «lo studente ritiene che in matematica si devono fare dei calcoli [...] e tende a far uso dei dati numerici presenti nel testo del problema per dare dunque una risposta formale, usando qualche operazione». Una minoranza della classe sostiene che la risoluzione di problemi matematici necessiti di riflessione e ragionamento, caratteristiche del problem solving, un solo bambino su 21 ha riscontrato una connessione tra problemi risolti in aula e problemi di vita quotidiana.

Nonostante tali concezioni sui problemi matematici, la quasi totalità del campione concorda sul fatto che attraverso la risoluzione di problemi matematici si accrescono le proprie competenze e, attraverso il ragionamento che il problema richiede, «si imparano cose nuove».

\subsection{Strategie di risoluzione di un problema}

Le domande riferite al secondo indicatore: "Analizzare le strategie attuate per la risoluzione di un problema matematico" avevano l'obiettivo di far emergere le modalità con cui gli allievi si avvicinano alla risoluzione di un problema matematico e le difficoltà che riscontrano durante la risoluzione.

Quasi la totalità degli alunni si rapporta alla situazione problematica evocando alla mente tutti i passaggi che sono soliti adottare. Si evidenzia come specificare il «fare ipotesi» nella procedura implichi nel ragionamento dei ragazzi che non tutti i problemi si eseguono tramite prassi già apprese, "a volte è la successione stessa delle operazioni risolventi a richiedere un atto strategico, talvolta creativo, da parte del solutore» (D'Amore \& Sbaragli, 2011, p. 95). Ne consegue che l'allievo ha ben chiaro di essere in una situazione nella quale è prevista la costruzione di una conoscenza, prerogativa del problem solving.

Per quanto concerne le difficoltà che gli studenti avvertono, interpretando le risposte date, salta all'occhio come percepiscano una fatica nell'argomentare, produrre e spiegare prove a sostegno di una loro tesi: ciò potrebbe essere correlato all'altro pensiero condiviso dagli alunni, cioè non capire quale operazione usare, come se implicitamente l'alunno desse per assodato che il fine ultimo del testo sia trovare un'operazione da formulare al fine di rispondere a una domanda e non sbagliare (sapendo che tutto ciò prima o poi condurrà a una valutazione che genera una sorta di ansia da prestazione). Un alunno riporta quanto segue: «ho difficoltà a spiegare il ragionamento perché se è giusto, è giusto poche volte e qualche volta lo spiego male». Molto probabilmente (bisognerebbe verificare ogni singolo problema che viene proposto), queste difficoltà nascono dalla difficoltà nel dare un senso al testo del problema e alla domanda correlata. Come sostiene Zan (2012b, p. 440), la domanda sia implicita che esplicita potrebbe «essere sul contesto, non nasce quindi nel contesto», andando a interrompere i legami tra i vari elementi della storia narrata e rendendo così confusionaria la comprensione del testo del problema.

\subsection{Emozioni e vissuti}

II terzo indicatore "Indagare i vissuti emotivi che emergono durante la risoluzione di un problema matematico" aveva l'intento di far emergere le emozioni e i sentimenti che i bambini provano, cercando anche di cogliere i motivi da cui scaturiscono determinate sensazioni.

Per 18 alunni, le emozioni vissute sono di tipo negativo; solo 3 alunni provano tranquillità. Ad esempio, una bambina riporta: "Mi sento un po' nervosa, un po' agitata perché penso che non ce la faccio, però ce la farò sicuramente».

La situazione è confermata anche dal grado che i bambini attribuiscono nella domanda 7 al senso di agio provato durante la risoluzione di un problema: di nuovo 18 alunni scelgono un punteggio minore o uguale a 3. Tali sensazioni sono dovute a un senso di insicurezza, alla paura di sbagliare e alla preoccupazione che ne deriva. Soffermarsi sulle emozioni vissute durante le situazioni di apprendimento è importante; la paura di sbagliare può creare un blocco nel singolo studente che nel tempo 
lo inibisce a rinforzare le strutture cognitive. Come ci viene ricordato, si riconosce:

«[...] nella nascita dell'emozione una componente cognitiva essenziale: l'emozione non è direttamente scatenata da un evento, ma dall'interpretazione di tale evento. [...] Se assumiamo questo punto di vista, non è l'esperienza matematica in sé che direttamente può scatenare emozioni negative, ma l'interpretazione che l'allievo ne dà, interpretazione che risente quindi delle sue convinzioni, dei suoi valori, dei suoi gusti e delle sue attitudini».

(Zan, 2007, p. 191)

Lavorare sull'aspetto motivazionale gioca quindi un ruolo fondamentale nel cambiamento delle percezioni degli alunni.

\subsection{L’argomentazione nei problemi matematici}

L'obiettivo del quarto indicatore "Indagare le competenze argomentative attuate per spiegare la procedura di risoluzione di un problema matematico" era quello di valutare, prima della somministrazione dei problemi scelti per la sperimentazione, quale fosse il livello individuale delle competenze argomentative in ambito matematico, invitando gli alunni a riflettere per iscritto su esperienze pregresse vissute.

Circa la metà degli studenti sostiene di spiegare i passaggi durante la risoluzione dei problemi, la motivazione che viene addotta si può ricondurre alla seguente risposta estrapolata dal questionario:

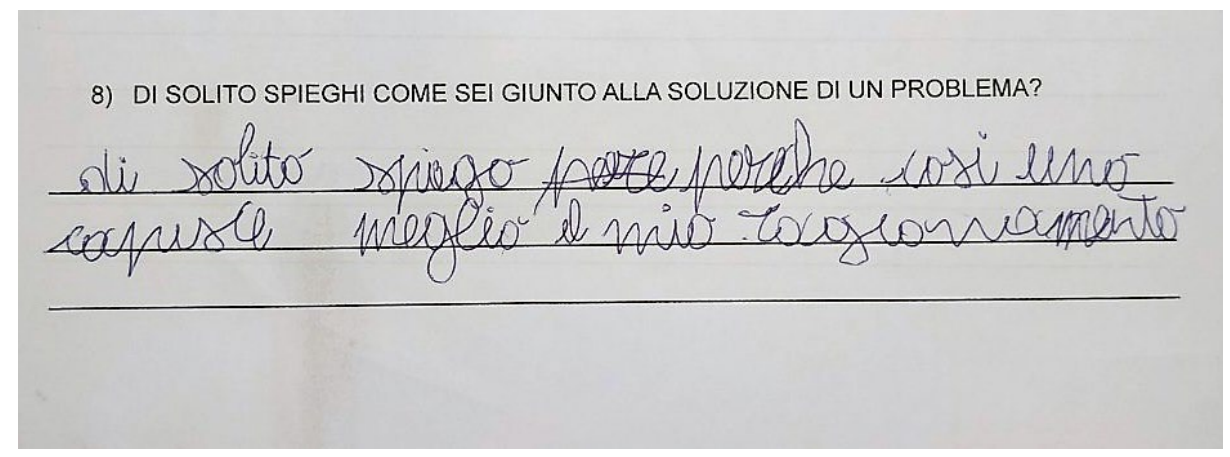

Figura 3. Estratto di un questionario.

Sebbene un bambino abbia dato una risposta non pertinente alla domanda fatta, più della metà della classe ritiene che argomentare i passaggi eseguiti per la risoluzione di un problema sia necessario per chiarire il percorso intrapreso dal lettore. Tre bambini riconducono la spiegazione alla sottolineatura di parole chiave, ancorandosi quindi a uno schema tradizionale di risoluzione di problemi basato sulla semplificazione del testo, adottando quelle che Zan (2012a, 2012b) definisce «scorciatoie cognitive»: inferendo direttamente sul testo le operazioni da fare, anziché rappresentarsi la situazione descritta e, su tale rappresentazione, costruire il processo risolutivo. I momenti in cui ciò accade non si limitano a situazioni in cui lo studente si trova da solo, la maggior parte della classe risponde alle domande 9 e 10 affermando che le discussioni, le argomentazioni sui procedimenti di risoluzione adottati avvengono quando si lavora in gruppo, e nei momenti di restituzione dei risultati, in linea quindi con gli obiettivi del Progetto ArAl e i principi del RMT. È importante, infatti, che processi cognitivi e competenze sociali possano svilupparsi in sinergia, facendo pratica della cooperazione positiva tra pari utilizzandola come risorsa anche per scoprire potenzialità e criticità di sé stessi all'interno di un contesto gruppale. 


\section{Problemi somministrati: i protocolli degli studenti}

Nei tre problemi somministrati, i bambini, suddivisi in gruppi, hanno trovato diverse strategie di risoluzione. In questo paragrafo si mostrano le strategie più significative sia per modalità di argomentazione sia per varietà di risoluzione.

\subsection{I protocolli del Problema n.1 - Caccia al tre}

In Figura 4 viene riportata la risoluzione a cui sono giunti tre gruppi ${ }^{8}$ (rispettivamente i gruppi n. 1, n. 3 e n. 4). Come si evince dall'argomentazione che accompagna la risoluzione del problema, i bambini hanno scritto tutta la successione dei numeri e hanno cerchiato i numeri contenenti la cifra 3; a ogni cifra trovata un altro bambino ha segnato una $X$ su un foglio attribuendone due al numero 33 , cerchiando la cifra 3 due volte. In questo modo sono giunti alla conclusione che la risposta esatta è 131.
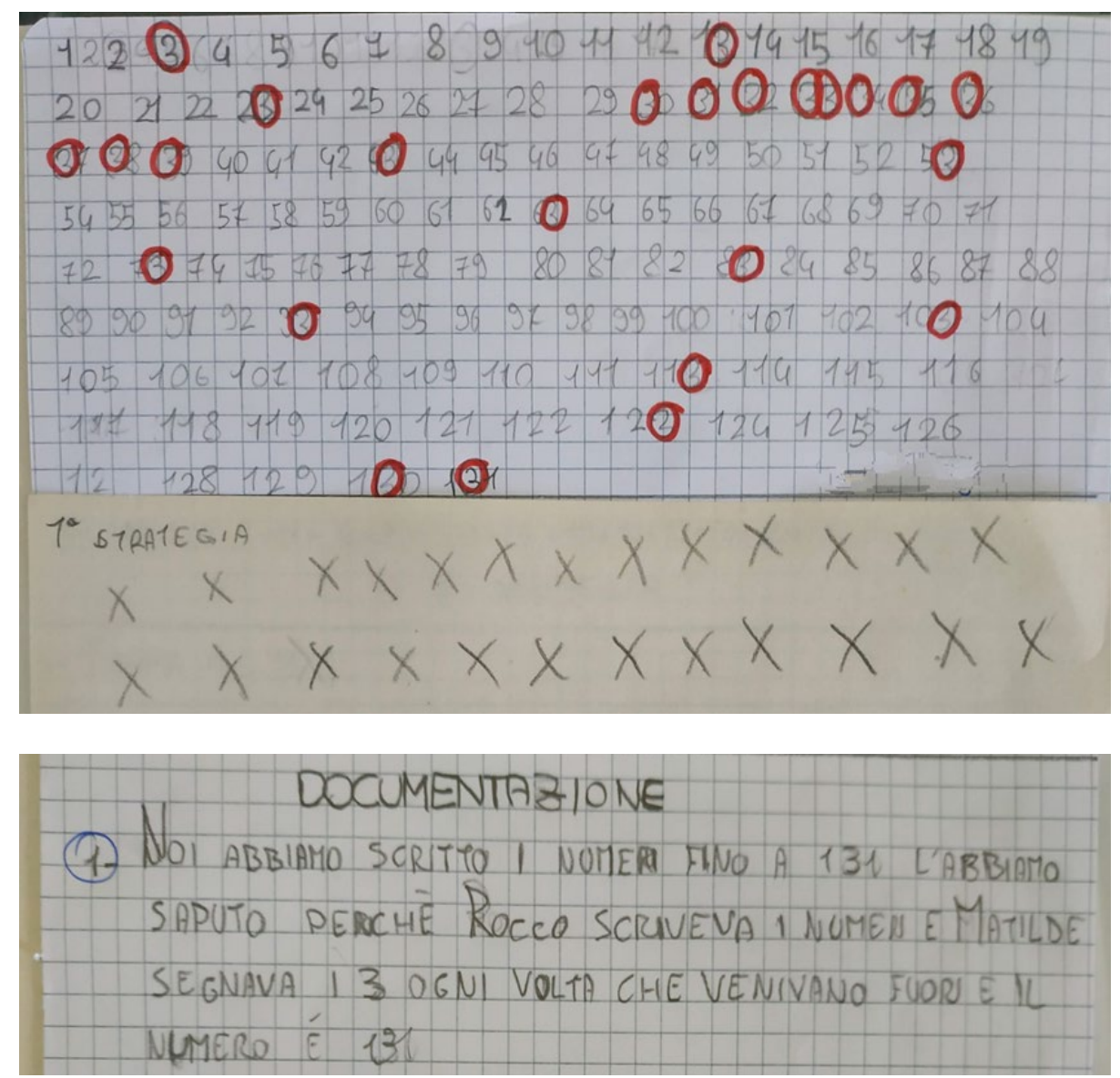

Figura 4. Esempio di protocollo, strategia n. 1.

Nella seconda variante (Figura 5), i bambini del gruppo n. 5 hanno riportato sul foglio soltanto i numeri contenenti la cifra 3 considerando mentalmente le decine, di volta in volta "saltando" tutti i numeri che non la contenevano.

8. I sette gruppi, come spiegato nel paragrafo "Metodologia", sono fissi; da qui in poi, quando si cita il numero di un determinato gruppo, ci si riferisce sempre allo stesso per composizione di alunni. 

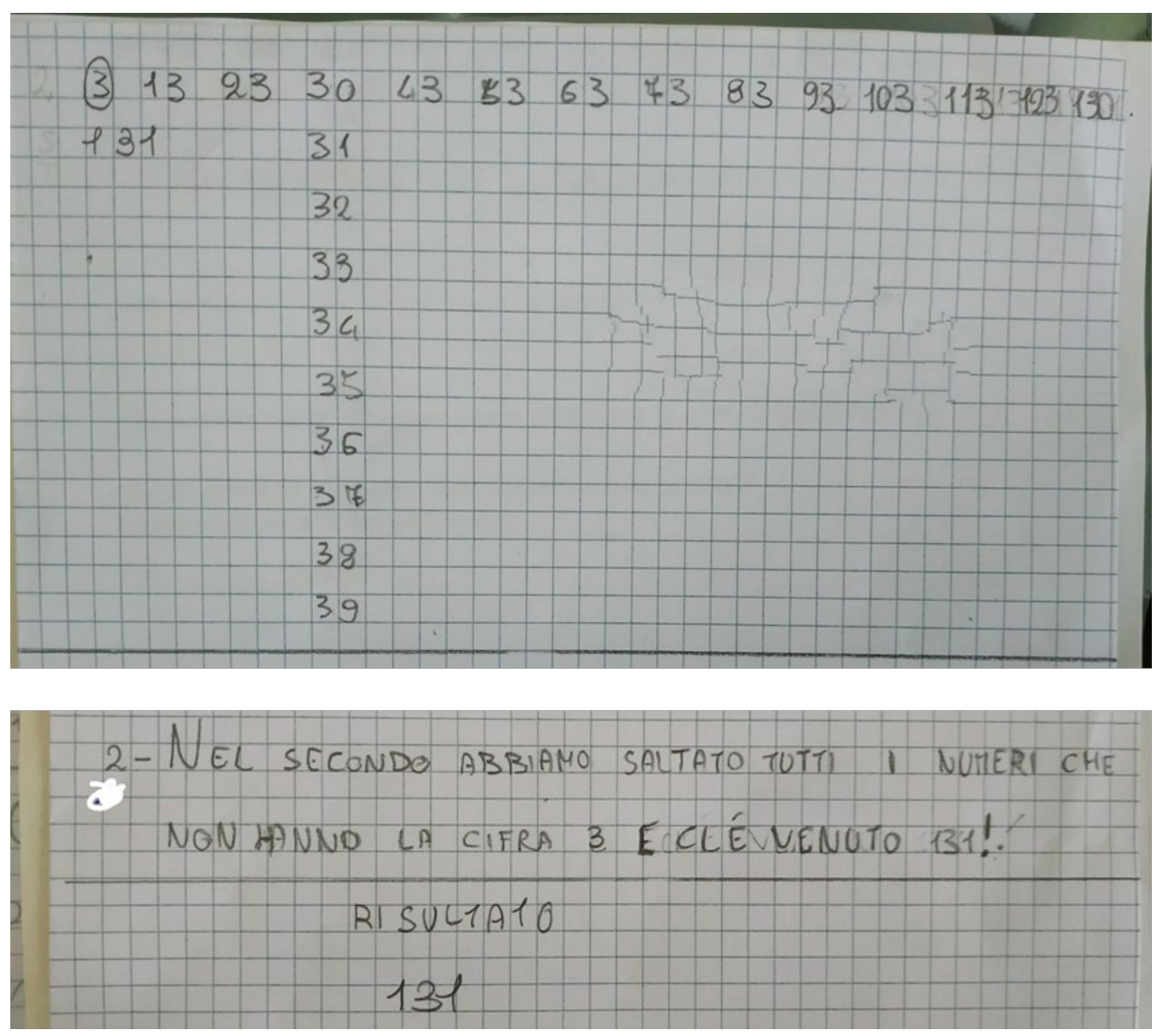

Figura 5. Esempio di protocollo, strategia n. 2.

Nella Figura 6, il gruppo n. 2 ha trovato una strategia (la terza) simile a quella della Figura 5: i bambini hanno scritto in successione soltanto i numeri che contenevano la cifra richiesta, cerchiando e contando di volta in volta la cifra 3 fino a giungere al numero 131. La differenza tra le due strategie risiede nel fatto che, nella precedente, è stato riportato per iscritto in modo più distinto il ragionamento fatto in base 10 .

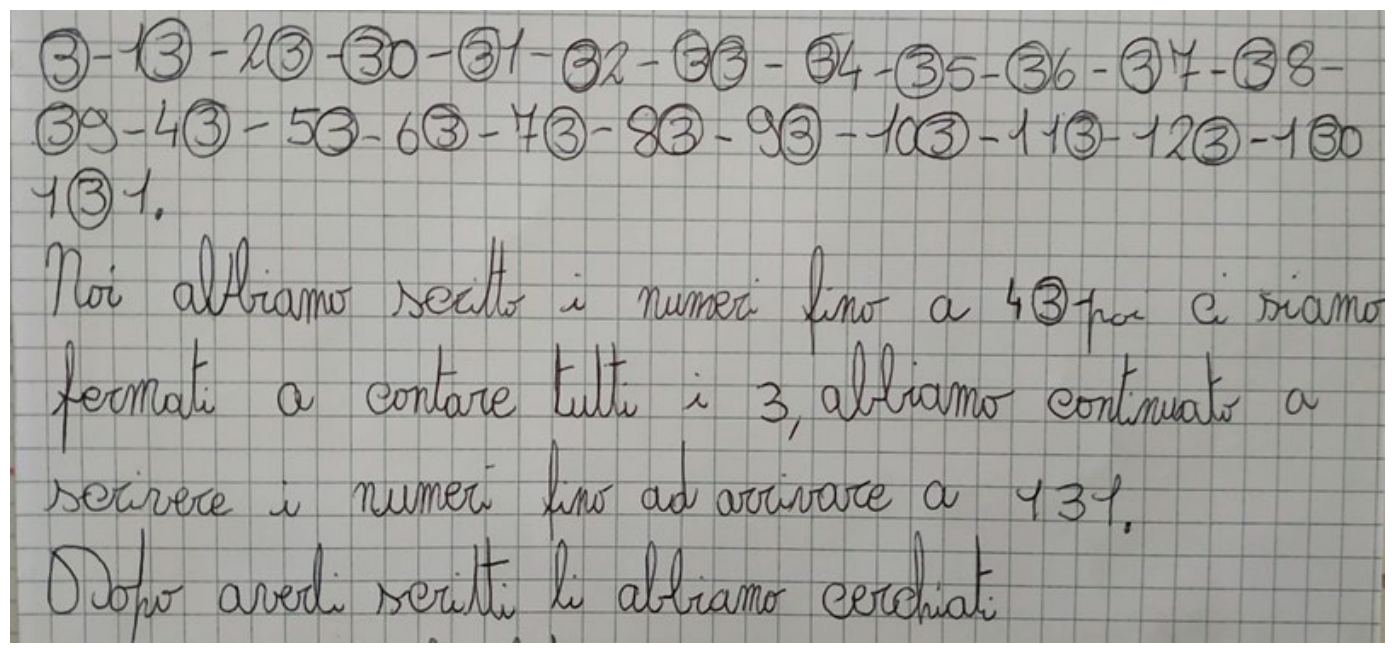

Figura 6. Esempio di protocollo, strategia n. 3. 
In Figura 7, il gruppo n. 6 ha usato una strategia che sembra simile alla precedente. L'argomentazione fornita dai bambini, tuttavia, chiarisce che la modalità di risoluzione è differente: i bambini dapprima hanno disegnato 24 cerchi per poi inserirvi di volta in volta i numeri contenenti la cifra 3. Resta però implicito che i bambini durante lo svolgimento abbiano considerato mentalmente le decine di volta in volta e abbiano contato la cifra 3 nel numero 33 due volte. Sebbene compaiano 24 cerchietti e non 25 , la soluzione trovata è giusta.

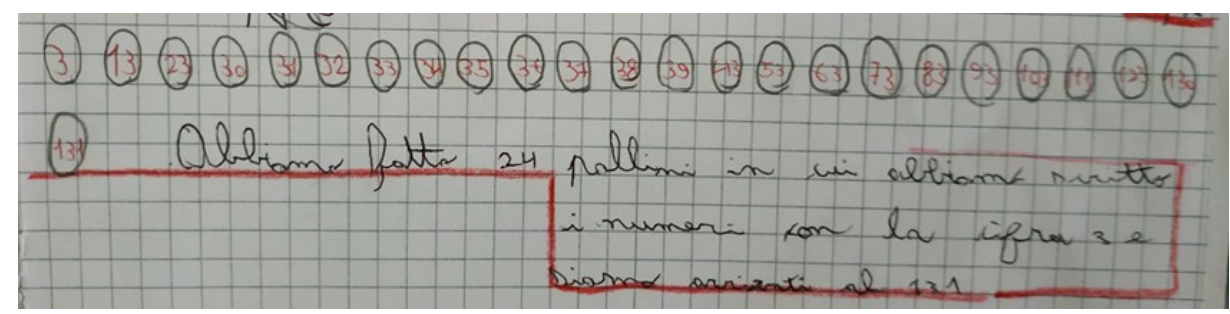

Figura 7. Esempio di protocollo, strategia n. 4

In Figura 8 il gruppo n. 7 ha creato una tabella numerata da 1 a 25. Dopo ha proseguito inserendovi i numeri contenenti la cifra 3 . Al numero 33 sono state riconosciute 2 frequenze di comparsa della cifra 3 rispettivamente alla settima e ottava posizione.

Leggendo l'argomentazione fornita dai bambini, si evince come essi implicitamente abbiano ragionato correttamente con uno schema, trovando come venticinquesimo numero 131; poi hanno cercato di spiegarlo a parole, riportando diversi tentativi di trovare un'operazione risolutiva che desse come risultato 131. In particolare, in un primo momento hanno moltiplicato 3 per 25 , e poi sommato ripetutamente 3 , ma il meglio che sono riusciti a ottenere è stato 132 . Nella ricerca di tale moltiplicazione, si può supporre che siano stati influenzati dall'aver interpretato l'espressione «venticinquesima volta» riportata nell'enunciato come "venticinque volte" e non come numero ordinale.

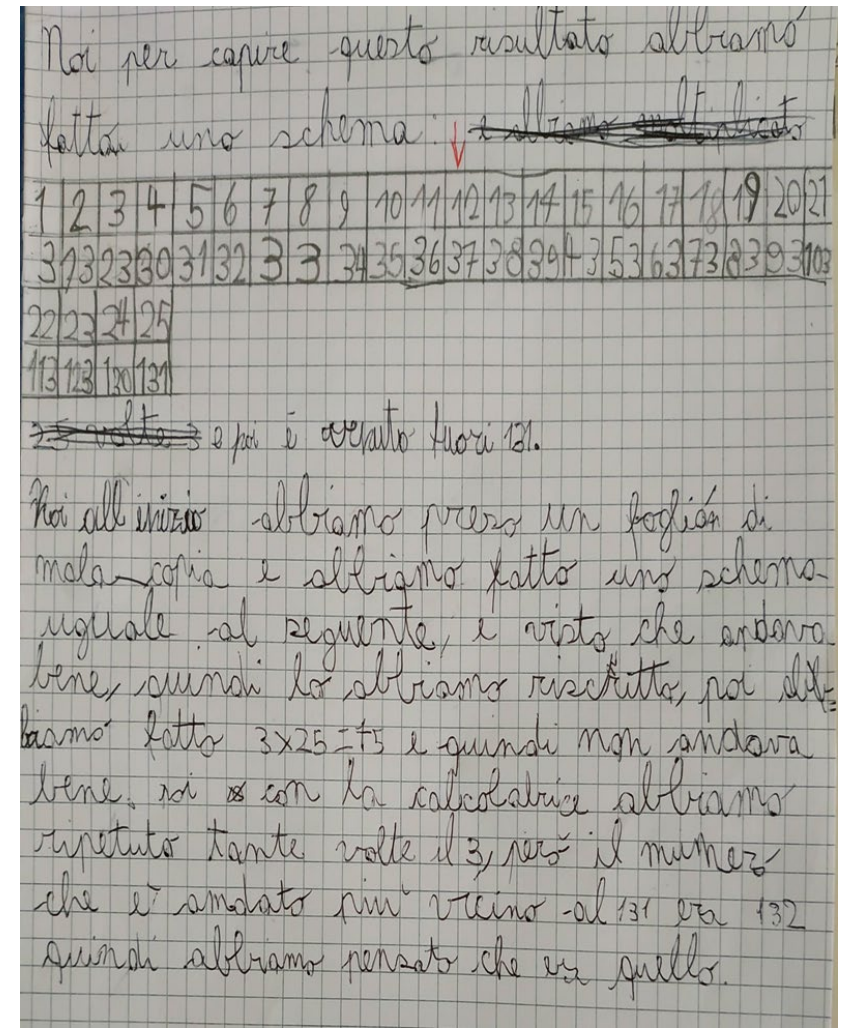

Figura 8. Esempio di protocollo, strategia n. 5. 


\subsection{I protocolli del Problema n. 2 - Tiro al barattolo}

Di seguito si riportano i protocolli più significativi inerenti al secondo problema somministrato "Tiro al barattolo". Sei gruppi su sette hanno trovato le tre combinazioni per risolvere il problema matematico (Figura 9).

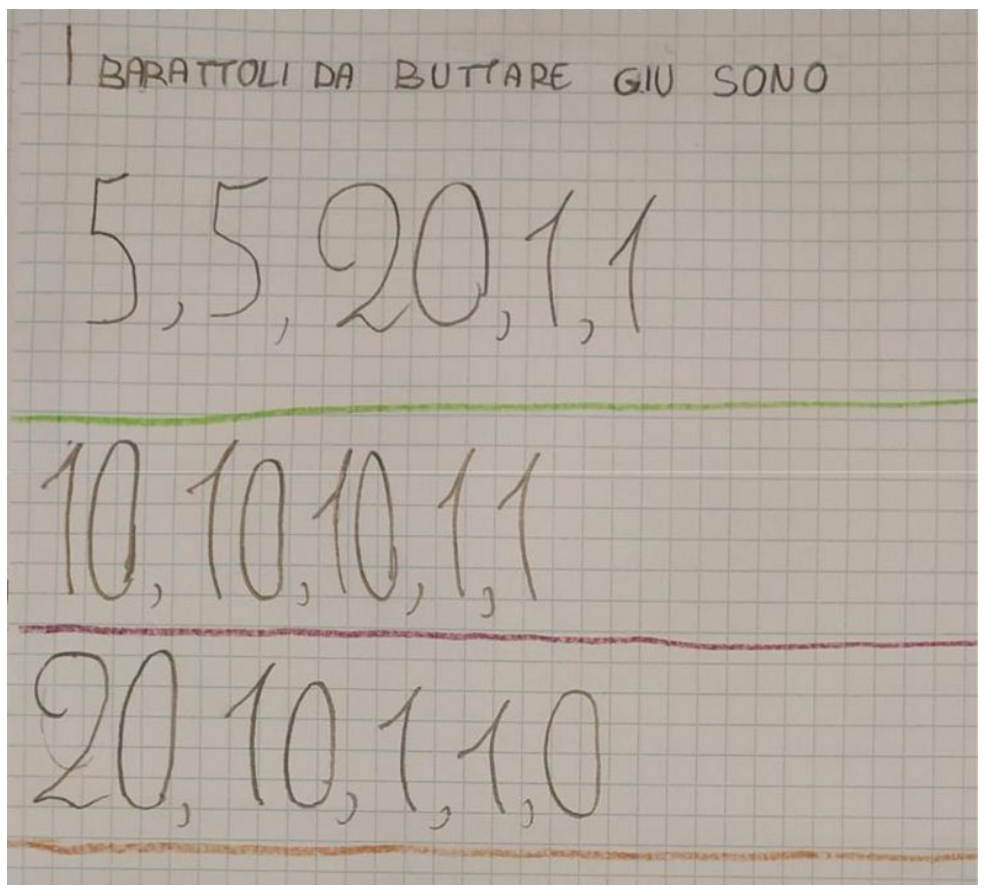

Figura 9. Combinazioni possibili.

Cinque gruppi (gruppo n. 2, n. 3, n. 5, n. 6, n. 7) su sette hanno utilizzato due argomentazioni differenti (Figure 10 e 11) ma tutte concordi nel considerare lo zero come possibile lancio sbagliato da conteggiare in una delle tre possibili combinazioni. Di seguito sono riportate le due argomentazioni più significative.

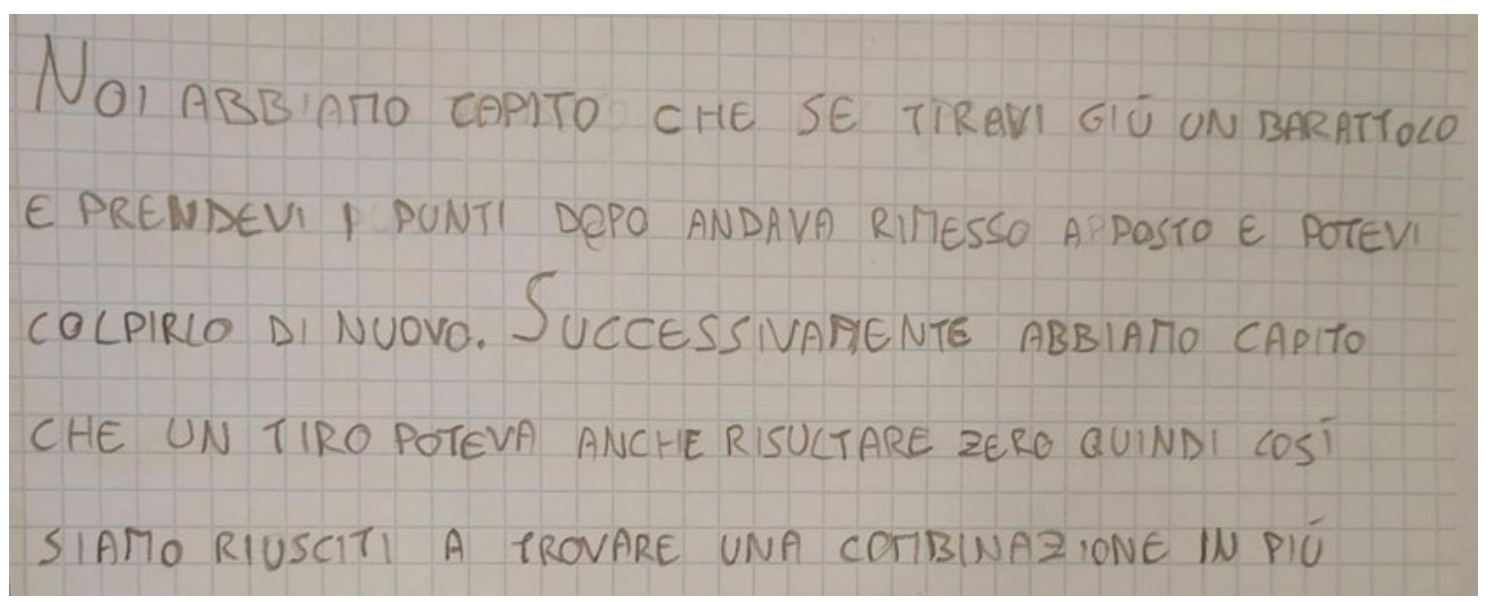

Figura 10. Argomentazione n. 1 


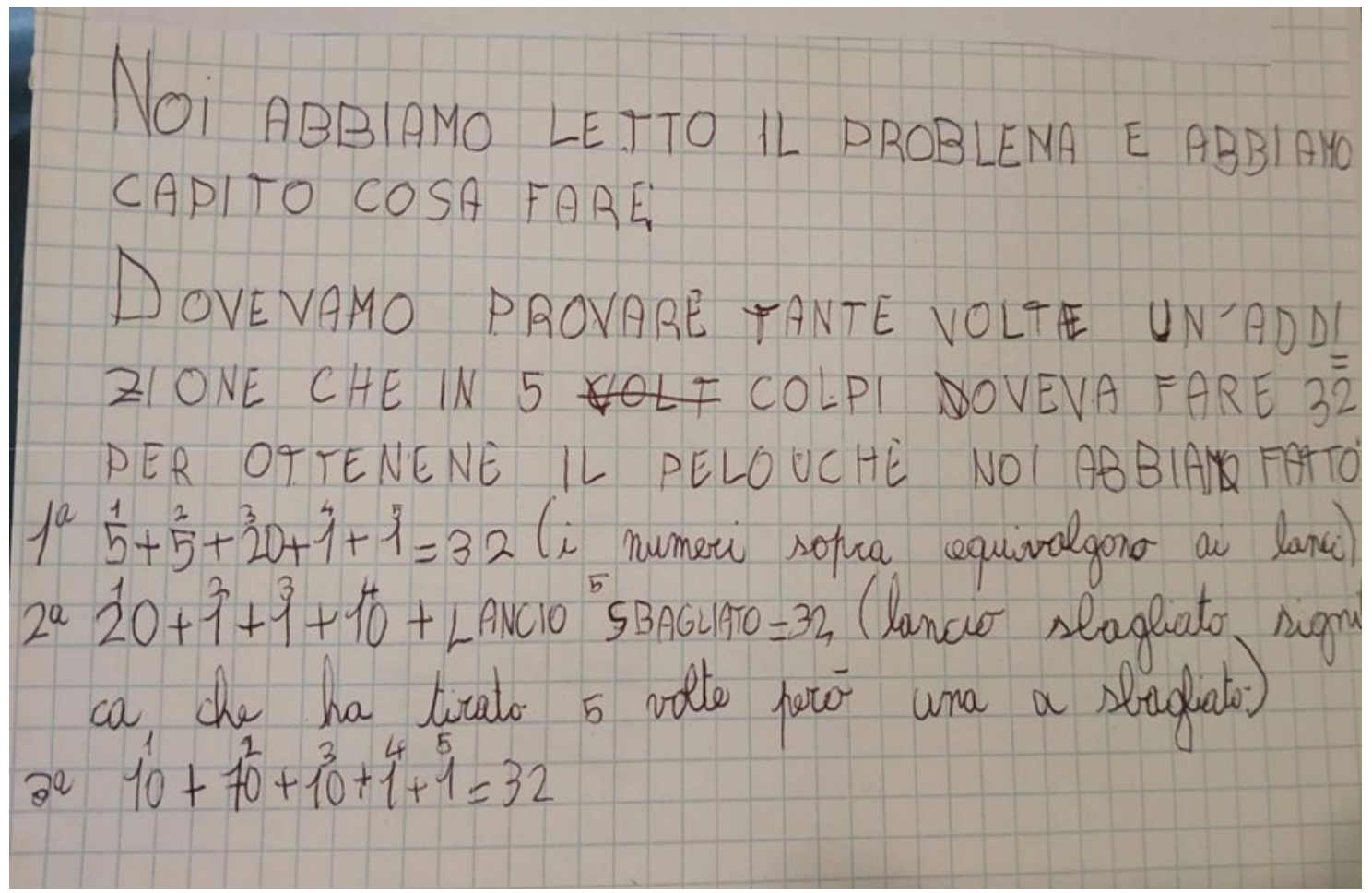

Figura 11. Argomentazione n. 2

Nella Figura 12, gli studenti del gruppo n. 1 hanno simulato graficamente la situazione narrata dal problema. II numero scritto sopra a ogni barattolo corrisponde a quante volte è necessario farlo cadere per giungere a 32, inoltre viene rappresentato graficamente con uno slash anche il tiro che ottiene zero punti, necessario per giungere alla terza combinazione possibile.

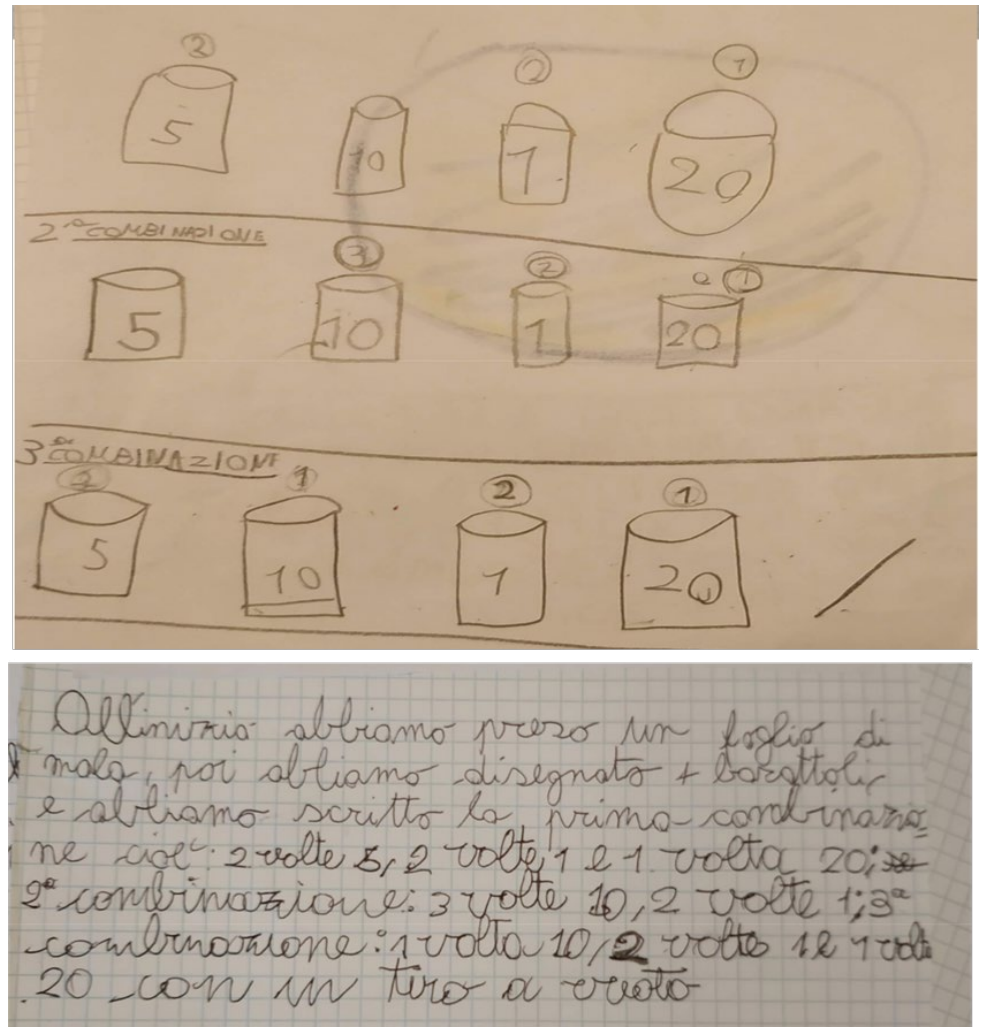

Figura 12. Esempio di protocollo, gruppo n. 1. 
Nella Figura 13, leggendo le argomentazioni, si può notare che il gruppo n. 4 non ha trovato la combinazione 10, 10, 10, 1, 1. Nei punti 1 e 3 (Figura 13) è riportata due volte la stessa combinazione ma con gli addendi in ordine diverso. Oltre a ciò, nel punto 1 è riportato il numero 0 nell'addizione numerica, mentre nel punto 3 compare l'espressione «nessun tiro» a cui non corrisponde l'addendo 0 nel calcolo scritto.

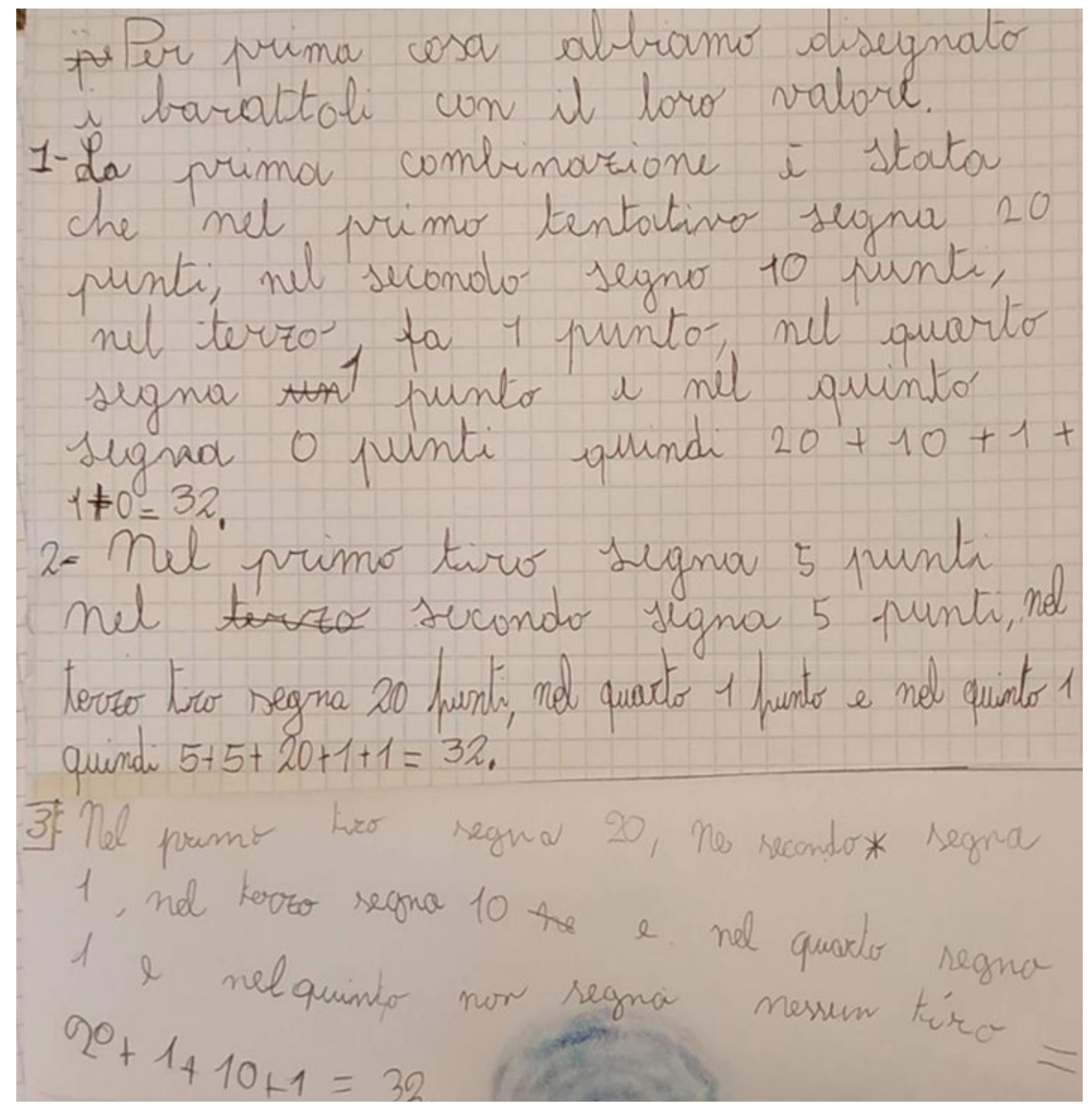

Figura 13. Esempio di protocollo, gruppo n. 4.

\subsection{I protocolli del Problema n. 3 - Il cuore di Martina}

Il terzo problema somministrato, "Il cuore di Martina", è attribuito solitamente dal RMT alle categorie 5 e 6 ovvero all'ultimo anno di primaria e al primo della secondaria di primo grado; ${ }^{9}$ per lo svolgimento sono previste delle conoscenze teoriche che si raggiungono in quei determinati anni scolastici. In accordo con l'insegnante della classe, si è deciso di somministrare ugualmente il problema per verificare se gli alunni sarebbero giunti ugualmente alla soluzione. Ne sono state trovate due diverse: la prima è stata applicata da sei gruppi su sette, la seconda è stata applicata e scoperta dal gruppo rimanente. 
Viene utilizzato il termine "scoperta" perché un gruppo ha applicato una strategia di risoluzione non presente nella banca dati dei problemi del RMT.

Di seguito (Figure 14, 15 e 16) vengono riportate e commentate le argomentazioni dei gruppi n. 2, n. 4 e n. 5, ritenute le più rilevanti e rappresentative della strategia $n .1$ (utilizzata anche dai gruppi n. 1 e n. 6). In tutte e tre le argomentazioni si nota che la procedura utilizzata è stata la medesima; i vari gruppi hanno deciso di ritagliare, nella figura a disposizione, la parte esterna al cuore e assemblarla, per poi constatare che le due aree sono equivalenti.

Nella seconda e terza argomentazione a sostegno della procedura, si può notare, prendendo come riferimento i principi teorici del progetto ArAl espressi nel par. 1.2, che vengono utilizzati dei termini afferenti al linguaggio specifico della matematica come «simmetrico» e «si equivalgono»; I'uso di tali termini denota una buona padronanza nell'utilizzo del linguaggio specifico, una volontà di superamento del "balbettio algebrico" rispetto alla prima argomentazione che utilizza il termine «uguali» tipico del linguaggio naturale.

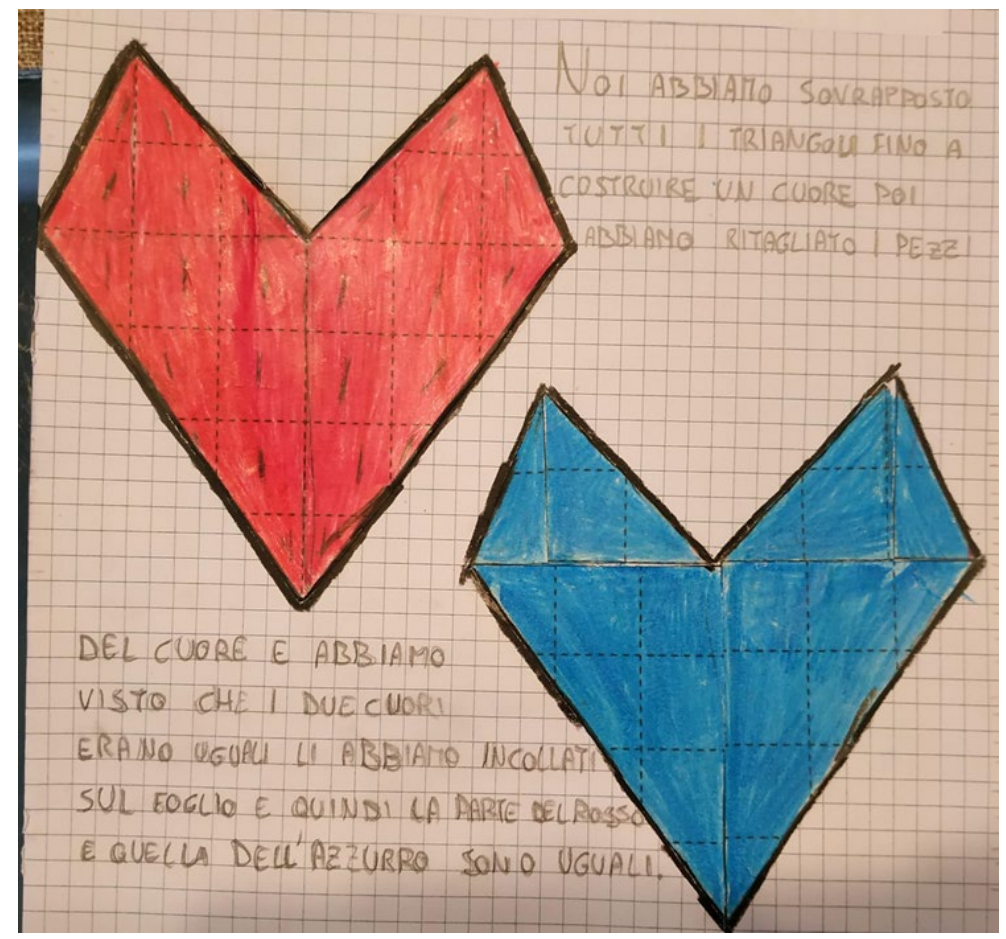

Figura 14. Argomentazione n. 1, gruppo n. 2

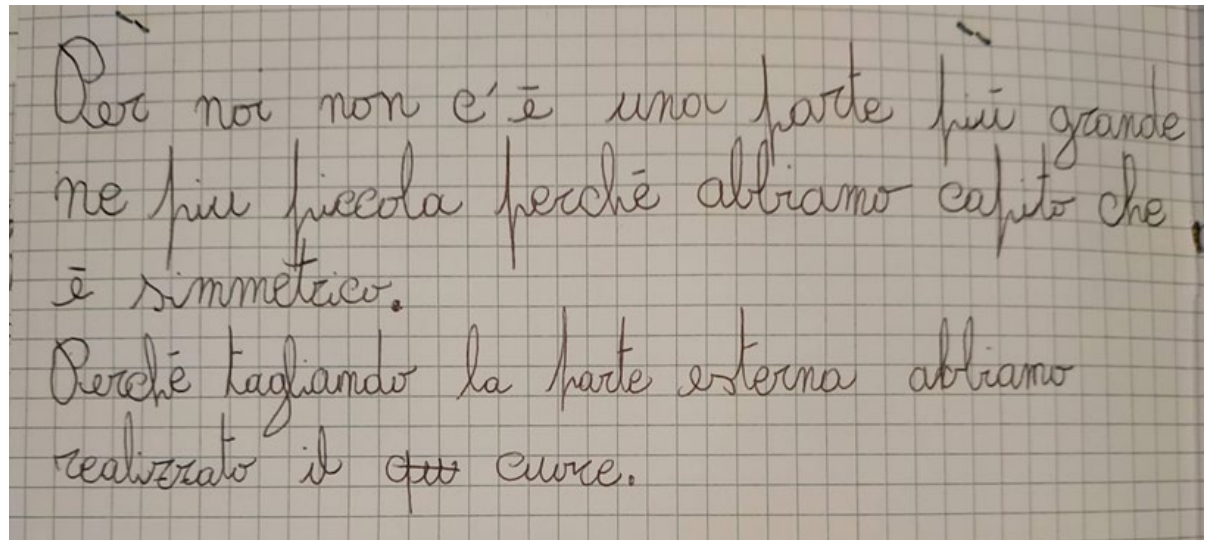

Figura 15. Argomentazione n. 2, gruppo n. 4. 


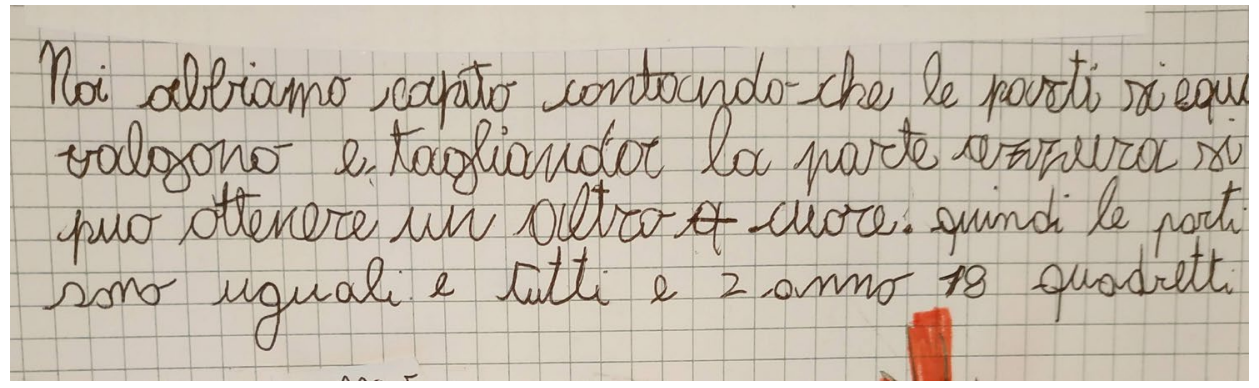

Figura 16. Argomentazione n. 3, gruppo n. 5

L'utilizzo di questa tipologia di problemi è un'occasione di valutazione in itinere per l'insegnante, che può verificare le diverse competenze degli alunni e la padronanza del lessico acquisito come nel caso sovraesposto. In particolare, con I'analisi dei risultati di questo problema, I'insegnante potrebbe verificare le conoscenze in possesso degli alunni e prevedere delle lezioni di approfondimento o di recupero, prima di introdurre nuovi concetti.

In Figura 17, viene riportata la strategia di risoluzione usata dal gruppo n. 3. Tale strategia è simile alla precedente, ma differisce per la modalità di ricostruzione del secondo cuore. I bambini hanno tagliato tutta l'area esterna al cuore e, nel ricomporlo, hanno usato come guida quello rosso, giungendo alla medesima conclusione.
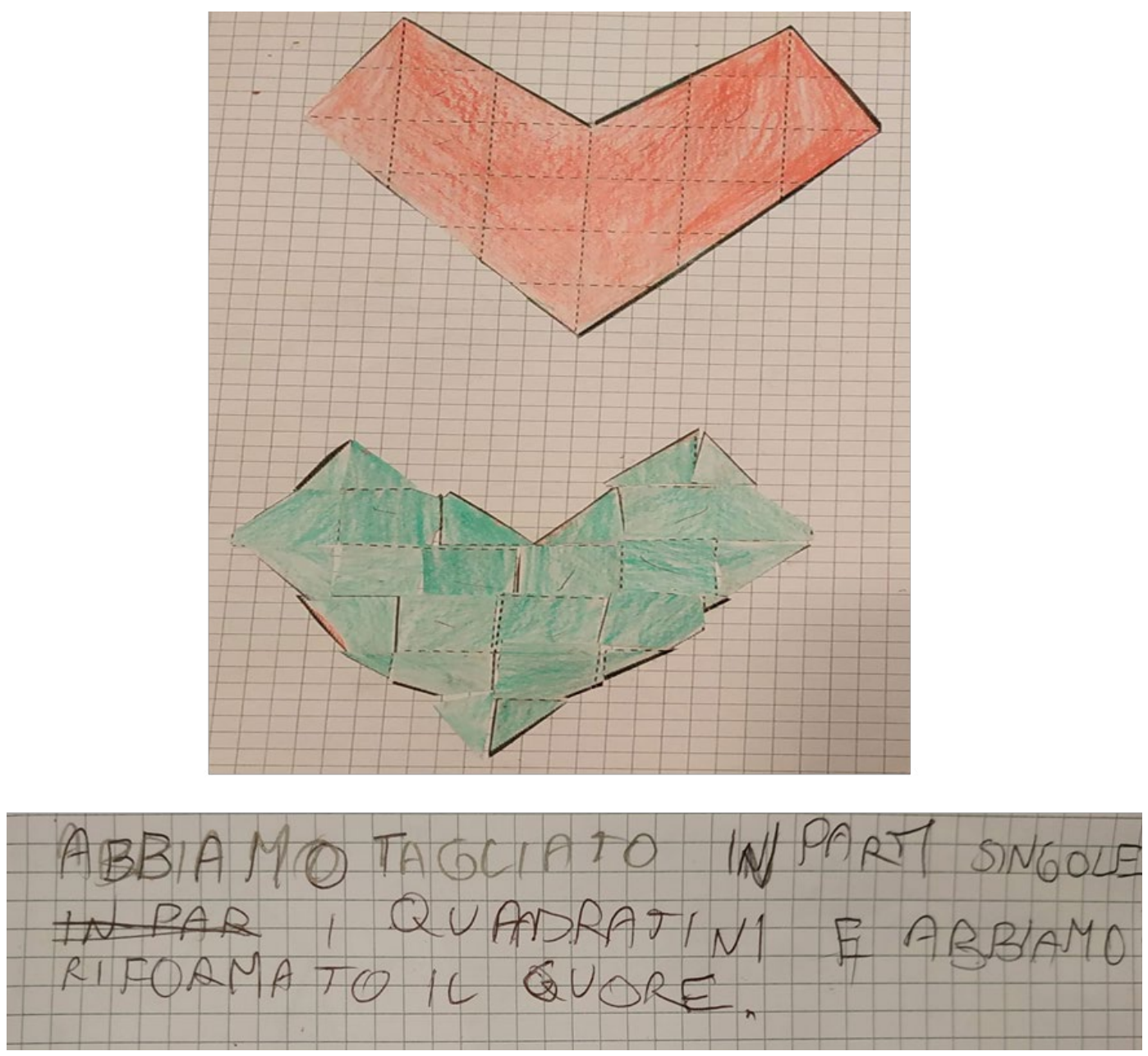

Figura 17. Esempio di protocollo, strategia n. 2. 
Si riporta infine il protocollo del gruppo $\mathrm{n}$. 7 che ha trovato una strategia di risoluzione differente (Figura 18).

I bambini di questo gruppo, per trovare la strategia di risoluzione, hanno applicato il pensiero computazionale: ragionando passo passo sulla strategia migliore per arrivare alla soluzione, si sono approcciati alla figura in modo algoritmico sia a livello di astrazione di pensiero che di linguaggio utilizzato. Nello specifico hanno utilizzato lo stesso numero e lo stesso colore per forme congruenti all'interno e all'esterno del perimetro del cuore. ${ }^{10}$ Terminata questa fase, hanno comparato il numero di pezzi interni ed esterni alla figura ottenuti. Rendendosi conto che ogni tipologia di forma aveva il medesimo numeri di pezzi, sono giunti alla conclusione che le due aree sono equivalenti.

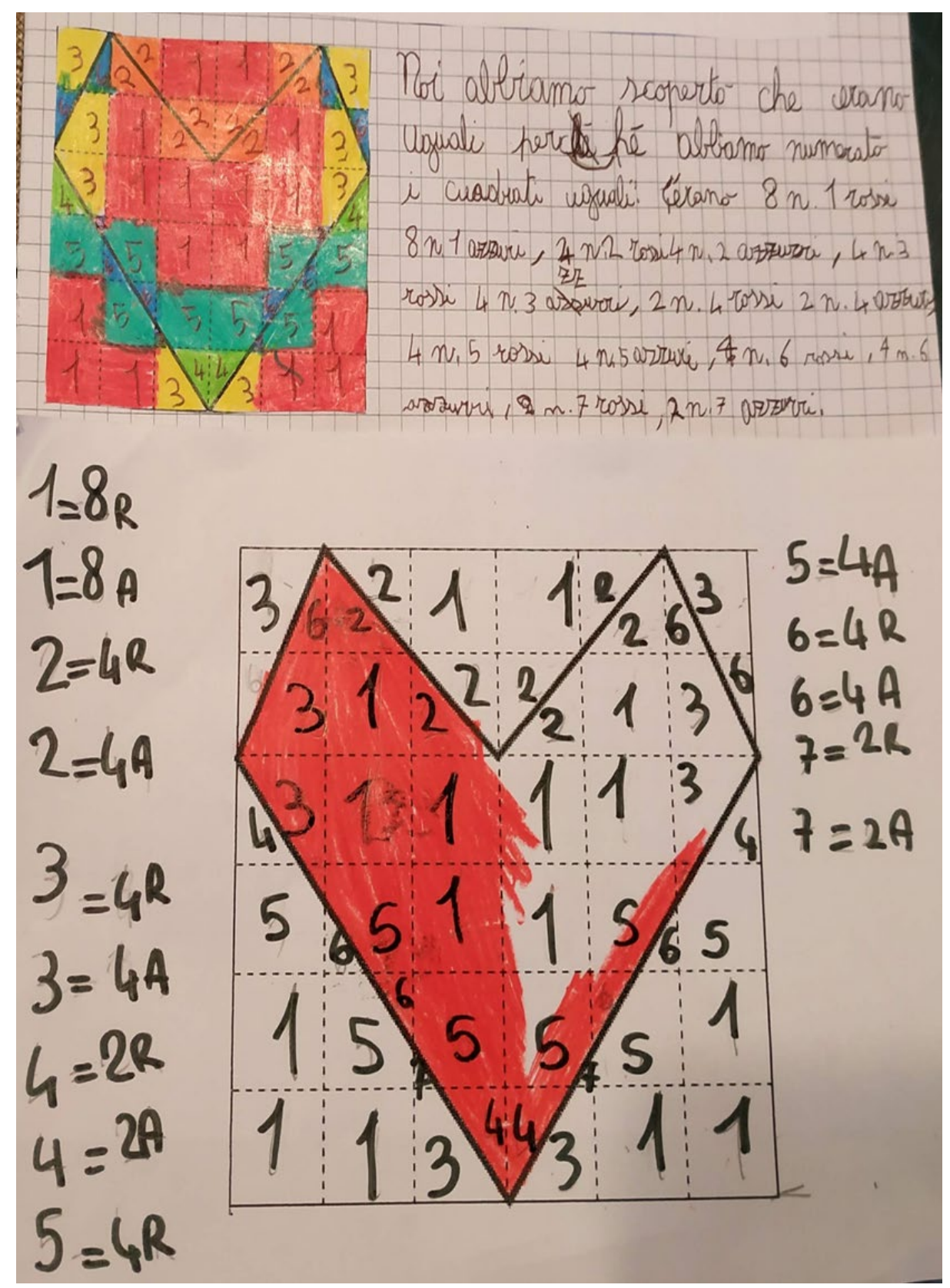

Figura 18. Esempio di protocollo, strategia n. 3.

10. Sebbene i bambini siano giunti alla risoluzione del problema, in realtà, il metodo non è applicato in modo formalmente corretto. Ad esempio, le figure segnate con il numero 3 o quelle segnate con il numero 6 non sono tutte tra loro equivalenti. 
In generale, tutti i bambini dei gruppi, pur non avendo nel proprio bagaglio le conoscenze delle formule geometriche per risolvere il problema, sono andati oltre: hanno tentato una strada nuova, come in un laboratorio di matematica, laboratorio inteso come momento «dove si costruiscono oggetti, si lavora concretamente, si ottiene qualche "cosa", è viva la tensione verso l'ideazione, la progettazione, la realizzazione di qualcosa di non ripetitivo, non banale» (D'Amore \& Marazzani, 2011, p. 16). I bambini hanno utilizzato un fare immaginato rendendolo concreto e operativizzabile sino a giungere alla risoluzione del problema.

\section{Questionario finale: analisi dei dati rilevati}

In questo paragrafo viene esposta I'analisi dei dati11 rilevati dal questionario somministrato dopo lo svolgimento dei tre problemi del RMT.

\subsection{Impressioni sull'approccio del RMT}

Le domande relative al primo indicatore "Indagare le concezioni personali sui problemi matematici seguendo la metodologia del RMT" puntavano a far emergere le prime riflessioni degli studenti su eventuali differenze rispetto allo svolgimento di problemi tradizionali.

Secondo 13 alunni vi è la possibilità di lavorare in gruppo e ne consegue maggior motivazione e coinvolgimento nella partecipazione al compito rispetto alla risoluzione tradizionale dei problemi.

Sebbene un bambino abbia dichiarato il desiderio di dare meno spazio ad attività didattiche incentrate sull'argomentazione, 7 bambini si ritengono soddisfatti delle regole di svolgimento del problema e non cambierebbero nulla.

Vengono anche forniti da 12 studenti diversi suggerimenti che possono fungere da spunti per la pratica didattica in aula:

- richiesta di tempi più dilatati: quando si inizia a lavorare sullo sviluppo della competenza argomentativa, è necessario dare ai bambini tempi più distesi per far propria la necessità e il focus sulla spiegazione della risoluzione del problema matematico;

- la richiesta di ampliamento del gruppo di lavoro: tale richiesta può essere interpretata come possibilità di discussione delle strategie da attuare assieme all'intero contesto classe;

- prevedere all'interno del complesso scolastico uno spazio specifico destinato ai laboratori matematici;

- come ultimo suggerimento, collegato ai due precedenti e collegato al problem solving, I'insegnante potrebbe calendarizzare con gli alunni un orario specifico da utilizzare nel laboratorio di matematica, uscendo dal solito contesto classe.

È importante tenere a mente che, quando ci si approccia ad attività di classe incentrate sulle metodologie descritte finora:

«[...] è naturale che i nostri studenti abbiano difficoltà nella maturazione di tali competenze, trattandosi di competenze complesse che costituiscono traguardi significativi di un percorso educativo lungo: proprio per questo bisogna dedicarci tempo e attenzione e progettare un percorso in verticale. Senza dimenticare che gli obiettivi relativi alle competenze di problem solving e argomentazione sono, per loro natura, obiettivi trasversali, che coinvolgono, nella loro specificità, molteplici discipline».

(Di Martino, 2017, p. 26)

$\overline{\text { 11. Le risposte complete degli alunni sono consultabili nell'Allegato } 2 .}$. 
I suggerimenti sopracitati, se introdotti nell'azione didattica, possono avviare un percorso per l'acquisizione di tali competenze.

\subsection{I ruoli nel contesto gruppo}

L'obiettivo del secondo indicatore "Individuare la ripartizione dei ruoli per risolvere il problema in contesto gruppale" era quello di indagare la ripartizione spontanea dei ruoli durante lo svolgimento dei problemi matematici.

Tra i principi del Progetto ArAl e del RMT, la cooperazione tra pari è un punto fondamentale per contrastare una logica competitiva e individualistica dell'apprendimento a favore di una di tipo democratico:

«Gli allievi devono dunque sapersi organizzare: devono dividere il lavoro fra i vari gruppi, gestire il tempo a disposizione, accettare i contributi di tutti, entrare nel punto di vista degli altri. Tali capacità non sono semplici da acquisire, ma sono sempre più indispensabili per adattarsi alla società attuale».

(Sito internet del RMT) $)^{12}$

Le risposte alle domande relative a questo indicatore ci forniscono informazioni dettagliate su come gli alunni abbiano lavorato per portare a termine il compito assegnato.

Dai risultati si evince che la maggioranza dei gruppi si è ripartita i compiti al suo interno lasciando aperta la possibilità di sperimentare altri ruoli laddove ve ne fosse la necessità, come dimostra questa affermazione:

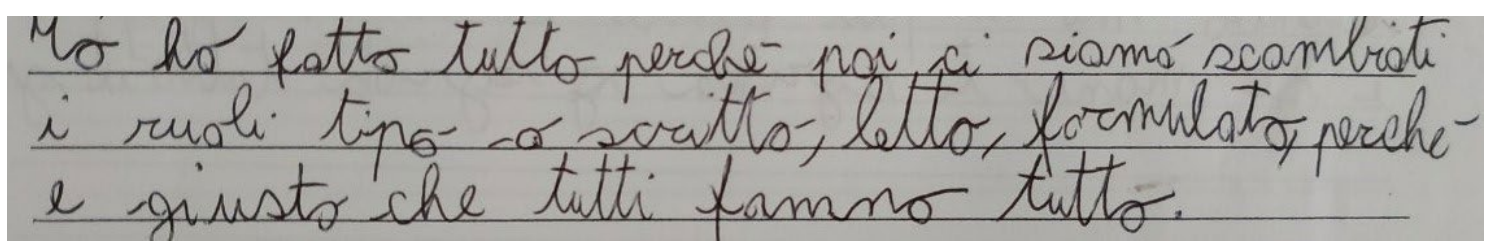

Figura 19. Risposta dal questionario

«L'apprendimento di gruppo rappresenta una strategia che ha come scopo finale quello di acquisire competenze cognitive e sociali. Ė dunque un percorso evolutivo, di progresso, che richiede una tempistica adeguata e uno sforzo da parte di chi è coinvolto» (Magnone, 2018, p. 84). Le strategie di tipo cooperativo rendono il bambino autonomo e consapevole del proprio contributo responsabile all'interno del gruppo.

\subsection{L'argomentazione nella risoluzione dei problemi}

Le domande del terzo indicatore "Evidenziare le strategie argomentative messe in atto per formulare la procedura di risoluzione del problema" avevano l'obiettivo di indagare come gli alunni si relazionano e attivano le proprie competenze per fronteggiare la necessità di un'argomentazione, indispensabile ai fini della risoluzione del problema.

Nelle risposte, 19 alunni hanno sottolineato come, dopo la discussione tra pari delle strategie risolutive da cercare, sia seguita in modo del tutto naturale l'argomentazione per iscritto del percorso fatto.

12. Si rinvia al par. 1 per la consultazione del link. 
Seppure con motivazioni diverse, la totalità dei bambini ha ritenuto importante la fase argomentativa della risoluzione di un problema, in particolare hanno evidenziato che attraverso l'argomentazione è più facile far capire a chi si ha di fronte il ragionamento seguito prima di giungere alla conclusione. C'è un aumento della motivazione ad apprendere che scaturisce dall'esplicitazione del percorso fatto tramite l'argomentazione. Infine, la consapevolezza di imparare una nuova competenza trasversale, spendibile anche in altri campi della vita personale, lascia una libertà di espressione da non sottovalutare: mantenendo inalterata la propria individualità, i bambini sentono la libertà di esprimersi perché si è in un contesto di rispetto reciproco, basilare per l'apertura verso I'altro, non si parla di compromesso, di ostacoli da superare ma di condivisione e co-costruzione delle conoscenze.

\subsection{Emozioni e vissuti}

Attraverso le risposte alle domande dell'ultimo indicatore "Rilevare i vissuti dei bambini durante la risoluzione in gruppo dei problemi matematici", è possibile fare emergere un confronto con le sensazioni e le emozioni espresse nel questionario in entrata, analizzate nel par. 3.3.

Se nel questionario in entrata la maggioranza degli alunni aveva dichiarato di provare emozioni negative durante lo svolgimento di problemi matematici tradizionali, dopo l'esperienza di risoluzione dei problemi del RMT la situazione è completamente ribaltata: ben 16 bambini hanno affermato di sentirsi a proprio agio attribuendo un punteggio tra 4 e 5, mentre 4 bambini hanno scelto il valore 3 e soltanto un bambino ha provato un senso di disagio assegnando un punteggio di 2.

Le motivazioni con cui i bambini accompagnano questo ribaltamento delle emozioni forniscono degli spunti di riflessione importanti per gli insegnanti: attraverso la cooperazione, il lavoro per raggiungere uno scopo comune, aumenta la stima verso sé stessi, al punto da sentirsi più preparati (7 alunni) e non sentire più il peso della paura di sbagliare perché il progetto di risoluzione è condiviso (4 alunni), con la possibilità di confrontarsi non essendo da soli di fronte al compito (6 studenti).

Anche le risposte relative alle emozioni provate sono nettamente cambiate rispetto al questionario in entrata: nella domanda del primo questionario, 18 allievi avevano riscontrato emozioni di tipo negativo e legate al senso di ansia, di insicurezza e alla paura di sbagliare.

Nell'analoga domanda 9 del secondo questionario, invece, 19 studenti hanno manifestato emozioni positive, quali felicità, senso di tranquillità e agitazione positiva, funzionale allo svolgimento del compito assegnato.

Dall'analisi di questi dati e dal confronto con quelli del primo questionario, è evidente come gli aspetti motivazionali, anche in campo matematico, siano strettamente correlati a quelli cognitivi. Come afferma Zan (2007, p. 184) «le variabili motivazionali sono ritenute l'aspetto energetico dei processi di auto-regolazione sottostanti le attività di problem solving». Da qui ci si collega a come lo studente si percepisce e percepisce la disciplina che ha di fronte:

«[...] le decisioni che un soggetto prende, i processi di auto-controllo che attiva avvengono all'interno della cornice delle sue convinzioni. Fra queste convinzioni appaiono particolarmente significative le convinzioni che l'allievo ha sulla matematica, le sue teorie del successo, le convinzioni che ha su di sé e in particolare la convinzione di "potercela fare"».

(Zan, 2007, p. 182)

I questionari hanno dimostrato come una percezione di tranquillità, un clima disteso, un senso di sicurezza, siano stati importanti per il lavoro in gruppo. 


\section{Conclusioni e spunti didattici}

Dallo studio effettuato emerge come i risultati della sperimentazione si allineino agli studi e alle ricerche recenti riguardanti lo sviluppo della competenza argomentativa in campo matematico.

La domanda posta a monte di questa sperimentazione ha voluto indagare se lo sviluppo e l'utilizzo della competenza argomentativa nella risoluzione di problemi matematici possa incidere a livello didattico in modo positivo nei processi partecipativi e motivazionali per gli alunni.

Dall'analisi globale del primo questionario, emergono i seguenti aspetti rilevanti: nonostante per la maggior parte dei bambini, almeno all'inizio della sperimentazione, un problema sia identificabile come tale quando prevede delle operazioni da trovare oppure ha delle domande a cui dare una risposta (tramite lo svolgimento di una serie di operazioni), la quasi totalità degli alunni riconosce come scopo dei problemi l'imparare cose nuove tramite il ragionamento e la riflessione. Ciò li spinge a leggere e rileggere un problema e fare ipotesi sulle possibili risoluzioni prima di delineare la strategia scelta.

Tra le difficoltà emerse per la risoluzione di problemi tradizionali, sono menzionate la fatica a esplicitare il ragionamento fatto e le difficoltà a decifrare il senso del testo. Ne consegue l'affiorare di sentimenti di tipo negativo per la maggior parte del campione, dovuti alla paura di sbagliare in primis e al senso di ansia e insicurezza provato successivamente.

Analizzando invece i dati rilevati dal secondo questionario, i primi elementi di differenza che emergono rispetto alla risoluzione dei problemi tradizionali riguardano la possibilità di condivisione del percorso per giungere alla risoluzione del problema matematico: ciò conduce gli alunni a trovare più motivante il contesto in cui sono calati al punto da spingerli a fornire dei suggerimenti didattici da implementare.

Dopo l'esperienza di discussione e argomentazione delle procedure di risoluzione, tutti gli alunni hanno concordato che argomentare è funzionale a rendere più comprensibile le scelte intraprese, permette di esperire una libertà di espressione che conduce anche a «imparare cose nuove», ampliando quindi il proprio bagaglio di conoscenze.

In ultima istanza, dai risultati dell'ultimo indicatore, è evidente come l'esperienza abbia ribaltato i vissuti emotivi dei bambini rispetto alla risoluzione di problemi: confrontando le risposte fornite tra il questionario in entrata e in uscita, si può affermare che l'utilizzo in classe di problemi che seguono gli obiettivi del Progetto ArAl e I'approccio didattico del RMT favorisce l'acquisizione di competenze in modo autonomo, I'assunzione di responsabilità da parte degli allievi, la visione critica e la messa in atto di strategie di condivisione del sapere in virtù di una crescita comune e una co-costruzione dei saperi. È importante però ricordare che il lavoro di regia dell'insegnante è necessario e basilare per l'apprendimento: è attraverso esso che si favorisce la costruzione dei saperi.

Utilizzare strategie didattiche, come quella sperimentata in aula, può avere ricadute positive sul contratto didattico: ad esempio, nel momento in cui l'insegnante fa in modo che lo studente comprenda gli errori fatti a causa di "misconcezioni" o di clausole del contratto didattico, senza che insorga in lui la paura di sbagliare. ${ }^{13}$

Si riportano, infine, alcuni aspetti emersi dall'analisi dei protocolli degli studenti. Ciò è funzionale ad avere una visione più dettagliata a sostegno dei risultati riscontrati.

Interessanti implicazioni didattiche emergono dal fatto che alcuni problemi, ad esempio "Il cuore di Martina", hanno coinvolto conoscenze non ancora trattate: nonostante il "deficit di sapere", tutti gli studenti hanno risolto il problema cimentandosi nella ricerca di soluzioni razionali e originali.

Un gruppo su sette ha utilizzato una strategia di risoluzione inedita, ricorrendo al pensiero computazionale: una volta appurato che la strategia trovata fosse giusta, è stata contattata la referente della

13. Per approfondire i concetti e gli studi relativi alle misconcezioni e al contratto didattico si rinvia agli studi di D'Amore e Sbaragli (2011). 
sezione Romagna del RMT, la quale ha chiesto di visionare la nuova strategia trovata per inserirla ufficialmente tra le possibili strategie di risoluzione nelle analisi a priori dei problemi del RMT.

Dal confronto tra le argomentazioni fornite per la strategia n. 1 del problema "Il cuore di Martina" (Figure 14, 15 e 16), si può osservare che l'argomentazione, soprattutto il tipo di argomentazione utilizzata, ha fatto emergere come alcuni gruppi hanno utilizzato un linguaggio specifico (termine «simmetrico») mentre altri hanno esposto la loro dissertazione aiutandosi con il linguaggio comune (termine «uguali»). L'insegnante può analizzare le risposte fornite per attivare strategie didattiche mirate al rinforzo del linguaggio specifico, affinché anche la parte di classe che è carente in tal senso possa arricchire il proprio bagaglio di conoscenze e superare, o ridurre, la fase di "balbettio algebrico". Significativo anche il protocollo n. 5 del problema "Caccia al tre" (Figura 8): in questo caso I'utilizzo dell'argomentazione è importante perché è emerso un iniziale errore che non sarebbe emerso nella sola parte procedurale (confondere il numero cardinale 25 con il numero ordinale venticinquesimo riportato nel testo); questo dato può essere utilizzato come spunto di riflessione dall'insegnante da rivolgere a tutta la classe.

Riallacciandoci alle emozioni vissute prima e dopo la somministrazione dei problemi, appare chiaro come il contesto gruppale e la responsabilità condivisa portino gli alunni a vivere l'esperienza con meno ansia e maggior coinvolgimento. L'allievo che è spronato a partecipare attivamente sarà motivato a migliorare il proprio operato e ciò andrà a ricadere sulla propria autostima, ma anche sul rapporto di collaborazione che instaura con i suoi pari.

Esperire situazioni didattiche come quelle del RMT potrebbe giovare al superamento di possibili conflitti e ostacoli in quanto l'incremento di motivazione è correlato alla manifestazione di maggior autostima dei bambini che si mettono in gioco.

Possiamo far riferimento a quanto riscontrato già più di vent'anni fa:

«[...] una delle maggiori difficoltà del rapporto insegnamento-apprendimento consiste in questo: I'insegnante dovrebbe convincere l'allievo e sé stesso che quel che si apprende, lo si apprende per la vita e non per il breve spazio di tempo legato ad una prova, ad una verifica, ad una qualche forma di valutazione».

(D’Amore, 1999, p. 386)

L'argomentare e l'ipotizzare, in particolare, sono propedeutici allo sviluppo delle competenze matematiche e all'individuazione di relazioni di passaggio dal linguaggio naturale al linguaggio specifico. L'attività argomentativa potrebbe essere definita come lo strumento più generale per poter costruire catene deduttive nel linguaggio naturale, seguendo le modalità del prevedere e dell'interpretare, e in questo senso come attività di avviamento alla dimostrazione.

L'attività argomentativa risulta funzionale non solo al monitoraggio delle reali concezioni degli allievi, ma anche alla configurazione di elementi su cui focalizzare la trasposizione didattica e alla messa a punto di percorsi educativi attenti anche ai vissuti emozionali.

A tal proposito è possibile evidenziare alcuni accorgimenti didattici atti a valorizzare la competenza argomentativa nella pratica d'insegnamento:

- dedicare maggiore spazio e tempo a momenti di lavoro condiviso affinché gli alunni possano mobilitare i processi di interpretazione e riflessione sulle strategie di risoluzione messe in atto;

- come suggerisce Di Martino (2017), scegliere problemi da proporre in aula con un'adeguata difficoltà, perché questo porta a un ventaglio più ampio di risposte al problema da parte dei ragazzi (come nel caso del problema "Il cuore di Martina"), accompagnate da argomentazioni che l'insegnante può utilizzare per avviare momenti di discussione;

- esplicitare e condividere i criteri di valutazione, rendere palesi le attese dell'insegnante per evitare fraintendimenti da parte degli allievi e affinché si sentano motivati e coinvolti nel processo di insegnamento-apprendimento; 
- prendendo ad esempio gli studi di Zan (2012a, 2012b), proporre lavori di inferenza sul testo dei problemi per condurre i bambini a riconoscere e cogliere le connessioni tra contesto (ricco di riferimenti al concreto) e domanda;

- prevedere, laddove possibile, uno spazio all'interno della scuola, dedicato al laboratorio di matematica, quale luogo privilegiato del fare (D'Amore \& Marazzani, 2011). L'insegnante, mantenendo il ruolo di regia in questo luogo, adotta una devoluzione a favore della presa di responsabilità da parte del bambino, vero protagonista in questo ambiente.

Attraverso questa esperienza didattica emerge la consapevolezza di quanto la scuola di oggi necessiti della figura di un insegnante che non si limiti a leggere e catalogare i fatti e i risultati che di volta in volta scaturiscono dal proprio agire didattico: integrando l'expertise professionale e didattico in modo continuativo, accompagnato da pratiche di riflessione sul proprio operato, si può puntare a una progettazione educativa intesa come «percorso di ricerca per eccellenza» (Vannini, 2009, p. 100).

Porre l'attenzione sui vissuti dei bambini, su come percepiscono sé stessi e su cosa provano di fronte alla richiesta di un compito matematico è importante per gli insegnanti: c'è la necessità nell'approccio valutativo di andare oltre le tracce che gli studenti lasciano trascritte a penna su un foglio. Affinché si possa delineare una didattica basata sull'agire, sul fare, sullo sviluppo delle competenze metacognitive e argomentative, è necessario indagare e partire dai vissuti emotivi dei singoli bambini, perché da essi si delineeranno i diversi approcci alla disciplina e gli apprendimenti che ne derivano.

È necessario che l'insegnante offra ai bambini occasioni per calarsi nella situazione problematica, attraverso la quale il bambino stesso, tramite la condivisione del percorso con i suoi pari e l'argomentazione quale mezzo atto a esternare i vissuti emotivi e i processi logici attuati, possa:

«[...] trovare una strada per uscire da una difficoltà, una strada per aggirare un ostacolo, per raggiungere uno scopo che non sia immediatamente raggiungibile. Risolvere problemi è un'impresa specifica dell'intelligenza e l'intelligenza è un dono specifico del genere umano: si può considerare il risolvere problemi come l'attività più caratteristica del genere umano».

(Polya, 1945, p. 130, traduzione dell'autore)

\section{Bibliografia}

D’Amore, B. (1993). Problemi. Pitagora Editrice.

D’Amore, B. (1999). Elementi di Didattica della Matematica. FrancoAngeli.

D'Amore, B., \& Marazzani, I. (2011). Problemi e laboratori, metodologie per I'apprendimento della matematica. Pitagora Editrice.

D’Amore, B., \& Sbaragli, S. (2011). Principi di base di didattica della matematica. Pitagora Editrice.

Di Martino, P. (2017). Problem solving e argomentazione matematica. Didattica della matematica. Dalla ricerca alle pratiche d'aula, 1, 23-37. https://www.journals-dfa.supsi.ch/index.php/rivistaddm/article/view/32

Magnone, S. (2018). II Rally matematico e la cooperazione tra allievi di scuola elementare. Didattica della matematica. Dalla ricerca alle pratiche d'aula, 4, 82-99. https://www.journals-dfa.supsi.ch/index.php/rivistadd m/article/view/53

Malara, N. (2009). Processi educativi per promuovere nelle classi un approccio costruttivo all'early algebra. Consapevolezze emerse negli insegnanti. http://www.progettoaral.it/wp-content/uploads/2016/08/Malara-attiNTSE-5-10-09.pdf 
Malara, N., \& Navarra, G. (2008). Analisi critica di processi di classe in ambito aritmetico-algebrico come modalità di formazione degli insegnanti. In O. Robutti (A cura di), Atti del III Convegno Nazionale Di.Fi.Ma. (pp. 164-170). Università di Torino.

Ministero dell'Istruzione, dell'Università e della Ricerca. (2012). Indicazioni Nazionali per il curricolo della scuola dell'infanzia e del primo ciclo d'istruzione. Annali della Pubblica Istruzione, Numero Speciale. http://www. indicazioninazionali.it/wp-content/uploads/2018/08/Indicazioni Annali Definitivo.pdf

Polya, G. (1945). How to solve it: a new aspect of mathematical method. Princeton University Press.

Vannini, I. (2009). La qualità nella didattica. Metodologie e strumenti di progettazione e valutazione. Edizioni Erickson.

Zan, R. (2007). Difficoltà in matematica. Osservare, interpretare, intervenire. Springer.

Zan, R. (2012a). La dimensione narrativa di un problema: il modello C\&D per I'analisi e la (ri)formulazione del testo (Parte I). L'insegnamento della matematica e delle scienze integrate, 35A(2), 107-126.

Zan, R. (2012b). La dimensione narrativa di un problema: il modello C\&D per l'analisi e la (ri)formulazione del testo (Parte II). L'insegnamento della matematica e delle scienze integrate, 35A(4), 437-467. 